\title{
On the Numerical Computation of Parabolic Problems for Preceding Times
}

\author{
By B. L. Buzbee* and Alfred Carasso**
}

\begin{abstract}
We develop and analyze a general procedure for computing selfadjoint parabolic problems backwards in time, given an a priori bound on the solutions. The method is applicable to mixed problems with variable coefficients which may depend on time. We obtain error bounds which are naturally related to certain convexity inequalities in parabolic equations. In the time-dependent case, our difference scheme discerns three classes of problems. In the most severe case, we recover a convexity result of Agmon and Nirenberg. We illustrate the method with a numerical experiment.
\end{abstract}

1. Introduction. Beginning with Hadamard, who drew attention to such problems, many analysts have been attracted to the study of improperly posed problems in mathematical physics. A recent survey by Payne in [22] lists over fifty references. Further references are to be found in [15], [16], [14], [3], [10], and [1]. The two best known examples of ill-posed problems are the Cauchy problem for Laplace's equation and the Cauchy problem for the backward heat equation. Some remarks concerning practical interest in such questions can be found in [7, p. 231], [15], [27] and [28].

From the viewpoint of numerical analysis, this ill-posedness manifests itself in the most serious way. We have discontinuous dependence on the data. Consequently [24, p. 59], every finite-difference scheme consistent with such a problem, and which is implemented as a marching process, is necessarily unstable. On the other hand, as was observed by John in [12] and Pucci in [23], continuous dependence can often be restored by requiring the solutions to satisfy a suitable constraint. Typically, one asks for nonnegative solutions or for solutions which satisfy an a priori bound, obtainable from physical considerations. The problem then is one of incorporating the constraint in the algorithm used for computing the solutions. One such successful method is the linear programming technique developed by Douglas in [9] and [10]. See also [4]. Computational experiments, using this method for the backward heat equation, have been described by Cannon in [3]. While quite good results are obtained in [3], it is not clear how one would extend this method to more general parabolic

\footnotetext{
Received November 3, 1971.

AMS (MOS) subject classifications (1970). Primary 65M30, 65M15, 35R25, 35K35; Secondary 65M20, 34G05, 35A35, 39A40.

Key words and phrases. Improperly posed problems, backward parabolic equations, timedependent coefficients, finite-difference scheme, jury-procedure, the backward beam equation, block Gaussian elimination, method of lines, variable domain operator, smoothing by growing diffusion coefficient, convexity inequalities for parabolic equations, long-time backward computation, matrix decomposition code.

* This author's work was supported by the U.S. Atomic Energy Commission.

** The work of this author was partly supported by the Sandia-UNM Research Project and NSF Grant GU-2582.
} 
mixed problems with time-dependent coefficients. In the above papers, essential use is made of the integral representation of the solutions in terms of the Green's function, and explicit knowledge of the latter seems to be necessary in order to perform the computations described in [3]. Another method is proposed by Douglas in [10] and Miller in [21], for problems where the solutions can be obtained by separation of variables. This method is based on expanding the given data in a truncated Fourier series, and requires knowledge of the eigenfunctions of the spatial operator.

In the domain of "general" linear parabolic problems, an interesting idea is discussed by Lattès and Lions in their recent book [14] along with several numerical experiments. This is the so-called "quasi-reversibility" or Q.R. method. Applied to the backward heat equation, this method consists in singularly perturbing the spatial operator by the addition of a higher-order term. The sign of the extra term is chosen so that the perturbed problem is well posed in the direction of decreasing time. Integrating backwards from the terminal data $u(x, T)$, one obtains an "initial-function" $u_{\epsilon}(x, 0)$. This function does not converge as $\epsilon \rightarrow 0$, as pointed out by the authors. It is one of infinitely many possible "initial functions". However, if it is used as initial data in the unperturbed forward problem, the corresponding solution $v_{t}(x, t)$ has the property that, at time $T, v_{\epsilon}(x, T) \rightarrow u(x, T)$ as $\epsilon \rightarrow 0$. Again, $v_{\epsilon}(x, t)$ does not converge if $t<T$. As the authors make clear, their aim is to solve a "control problem" associated with parabolic equations rather than approximate the solutions of the backward problem.

In this paper, we develop a finite-difference scheme for computing the solutions of linear selfadjoint parabolic problems backwards in time, given an a priori bound $M$ on the solutions, and given the terminal data to a known accuracy $\delta$ in the $L^{2}$ norm. We make no hypotheses regarding the "power spectrum" of either the terminal data or its error component. (See [28], [29].) Moreover, explicit knowledge of the analytic solution operator is not required. The scheme is applicable to problems with variable coefficients depending on time and is implemented as a "jury" procedure rather than a time-marching method. It is based on the "backward beam equation" previously discussed in [5] and [6]. In the case of the continuous backward problem, the uncertainty in any of its solutions can be bounded in terms $M$ and $\delta$, using certain convexity results. See for example [25] and [11, p. 182]. The error bounds in our difference scheme differ from this fundamental uncertainty only by the contribution due to truncation. The time-dependent case turns out to be rather interesting in this connection.

In Section 2, we develop some preliminary results associated with the backward beam equation. In Section 3, the connection between parabolic problems and the backward beam equation is explained. There, we write down the difference scheme as it applies to second-order parabolic problems in rectangular regions, in two space dimensions, with Dirichlet boundary conditions. More complicated problems can also be treated. For expository reasons, the discussion in Section 3 is not specifically oriented towards the backward problem. The latter is considered in Section 4, where the previous results are tied together. Section 4 contains the main results of the paper. Finally, in Section 5, we describe the results of a computational experiment on a one-dimensional problem whose exact solution is known. More extensive calculations on two-dimensional problems, together with a discussion of methods for solving the difference equations, will appear in a later report. 
Because this paper is rather long, we offer the following guide to the reader. It is probably best to first skim through Section 2, and then proceed to Section 3 . The main result of Section 2 is motivated in the remark following Theorem 1. Since most of the machinery is developed in the previous sections, the exposition in Section 4 is unhindered. Thus, Section 4 is the easiest one to read.

While this work was in progress, we had the pleasure of the advice and encouragement of many people. We particularly wish to thank R. Hersh, P. D. Lax, S. V. Parter, R. D. Richtmyer and Joel Spruck.

2. The Abstract Backward Beam Equation. In this section, we develop some preliminary results relating to an abstract situation, namely, a two-point problem for an ordinary differential equation in Hilbert space. The main result of this section, Theorem 1 below, will play a major role in Sections 3 and 4.

Let $H$ be a separable Hilbert space with scalar product $\langle\cdot, \cdot\rangle$ and corresponding norm $\|\cdot\|_{H}$. For each $t$ in the finite interval $[0, T]$, let $A(t)$ be an unbounded linear operator, which is closed and with domain $D_{A}(t)$ dense in $H$. In general, the domain of $A(t)$ will vary with $t$. We assume $A(t)$ to have the following property.

There exists a real number $\beta$ independent of $t$ such that $[A(t)+\lambda]^{-1}$ exists and is a bounded operator on $H$ whenever $\operatorname{Re} \lambda>\beta$. Furthermore,

$$
\left\|(A(t)+\lambda)^{-1}\right\|_{H} \leqq \frac{1}{\operatorname{Re} \lambda-\beta}, \quad \operatorname{Re} \lambda>\beta
$$

In the terminology of $[13$, p. 279], this means that, for each $t, A(t)+\beta$ is " $m$ accretive." Hence, in particular,

$$
\operatorname{Re}\langle A(t) v, v\rangle \geqq-\beta\langle v, v\rangle \quad \forall v \in D_{A}(t) .
$$

Consider now the problem

$$
\begin{aligned}
u_{t t}-A(t) u & =g(t), \quad 0<t<T, \\
u(0) & =f_{1}, \quad u(T)=f_{2},
\end{aligned}
$$

where $f_{1}$ and $f_{2}$ are given vectors in $H, u(t)$ and $g(t)$ are $H$-valued functions on [0,T], and $g(t)$ represents a forcing term. The above system was studied by one of us in [5] and applied to parabolic problems in [6]. In the above papers, it was assumed that

$$
\operatorname{Re}\langle A(t) v, v\rangle \geqq 0,
$$

rather than (2.2). Leaving aside the question of existence of solutions in (2.3), we have

Lemma 1. Let $\pi^{2} / T^{2}>\beta$. Then, there is at most one twice continuously differentiable solution of (2.3), (2.4).

Proof. Let $u$ and $v$ be any two solutions. Put $w=u-v$. Then,

$$
\begin{gathered}
w_{t t}-A(t) w=0, \quad 0<t<T, \\
w(0)=w(T)=0 .
\end{gathered}
$$

Hence 


$$
\begin{aligned}
\operatorname{Re} \int_{0}^{T}\left\langle w_{t t}, w\right\rangle d t & =\operatorname{Re} \int_{0}^{T}\langle A(t) w, w\rangle d t \\
& \geqq-\beta \int_{0}^{T}\|w(t)\|_{H}^{2} d t
\end{aligned}
$$

Integrating by parts, using (2.7), we get

$$
\frac{\pi^{2}}{T^{2}} \int_{0}^{T}\|w(t)\|_{H}^{2} d t \leqq \int_{0}^{T}\left\|w_{t}\right\|_{H}^{2} d t \leqq \beta \int_{0}^{T}\|w(t)\|_{H}^{2} d t,
$$

and the result follows.

Consider now the following finite-difference approximation to the system (2.3), (2.4),

$$
\begin{gathered}
\left(v^{n+1}-2 v^{n}+v^{n-1}\right) / \Delta t^{2}-A^{n} v^{n}=g^{n}, \quad n=1,2, \cdots, N, \\
v^{0}=f_{1}, \quad v^{N+1}=f_{2} .
\end{gathered}
$$

Here, $\Delta t=T /(N+1)$ is a small increment in the $t$-variable, $A^{n}$ denotes $A(n \Delta t)$, and, for each $n, v^{n}$ is an element of $H$ which presumably will approximate $u(n \Delta t)$, if the latter exists. Introduce the following notation. With $T=(N+1) \Delta t$, let $H^{N}(\Delta t)$ be the complex vector space of all $N$-tuples $\left\{v^{1}, v^{2}, \cdots, v^{N}\right\}$ where $v^{k} \in H$ for each $k=1,2, \cdots, N$. Elements of $H^{N}$ will be denoted by capital letters and represented as column vectors

$$
V=\left\{v^{1}, v^{2}, \cdots, v^{N}\right\}^{T}
$$

Equip $H^{N}(\Delta t)$ with the scalar product

$$
(V, W)=\Delta t \sum_{n=1}^{N}\left\langle v^{n}, w^{n}\right\rangle
$$

and write

$$
\|V\|_{H^{N}}=(V, V)^{1 / 2} .
$$

Since $H$ is separable, so is $H^{N}$. We will also use $N \times N$ matrices whose entries are linear operators in $H$. Such matrices represent linear operators in $H^{N}$. For any such matrix $Q$, we define

$$
\|Q\|_{H^{N}}=\operatorname{Sup}_{\|V\| H_{H^{N}=1}}\left\{\|Q V\|_{H^{N}}\right\},
$$

the supremum being taken over all $V$ in the domain of $Q$. We can now write the system (2.10), (2.11) as a single operator equation in $H^{N}$ namely,

$$
Q V=F-G,
$$

where $V$ is the vector in (2.12),

$$
\begin{aligned}
& F=\left(1 / \Delta t^{2}\right)\left\{f_{1}, 0, \cdots, 0, f_{2}\right\}^{T}, \\
& G=\left\{g^{1}, g^{2}, \cdots, g^{N}\right\}^{T},
\end{aligned}
$$

and $Q$ is the $N \times N$ tridiagonal matrix 


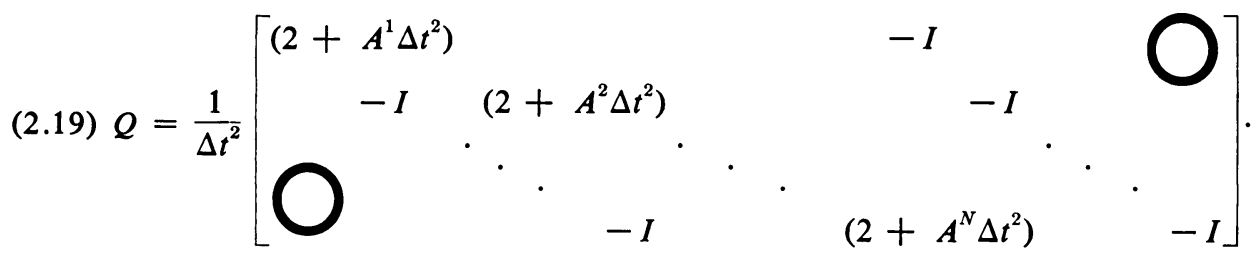

Note that in order for $V$ to be a solution of (2.16), it is necessary that the $j$ th component of $V$ belong to $D_{A}(j \Delta t)$ for each $j=1, \cdots, N$. Concerning the existence of solutions of (2.16) and the "stability" of this difference approximation, we have

THEOREM 1. Let the family $\{A(t)\}$ satisfy (2.1) with $\beta>0$ and let $T$ satisfy

$$
\pi^{2} / T^{2}>\beta \text {. }
$$

Then, for all sufficiently small $\Delta t$, there is a unique solution of (2.16) for arbitrary $F, G \in H^{N}$. Moreover, the following estimate holds:

$$
\begin{aligned}
\left\|v^{n}\right\|_{H} \leqq & \frac{\pi T^{3 / 2}}{\left[(1-\epsilon) \pi^{2}-\beta T^{2}\right]}\|G\|_{H^{N}}+O(\Delta t)\left\{\left\|f_{1}\right\|_{H}+\left\|f_{2}\right\|_{H}\right\} \\
& +\frac{\operatorname{Sin} \beta^{1 / 2}(T-n \Delta t)}{\operatorname{Sin} \beta^{1 / 2} T}\left\|f_{1}\right\|_{H}+\frac{\operatorname{Sin} \beta^{1 / 2} n \Delta t}{\operatorname{Sin} \beta^{1 / 2} T}\left\|f_{2}\right\|_{H},
\end{aligned}
$$

as $\Delta t \downarrow 0$, where $\epsilon>0$ is sufficiently small such that $(1-\epsilon) \pi^{2}-\beta T^{2}>0$.

If $\beta \leqq 0$ in (2.2), there is no restriction on $T$. With $\beta=0$, we have

$$
\left\|v^{n}\right\|_{H} \leqq \frac{T^{3 / 2}}{(1-\epsilon) \pi}\|G\|_{H^{N}}+\frac{(T-n \Delta t)}{T}\left\|f_{1}\right\|_{H}+\frac{n \Delta t}{T}\left\|f_{2}\right\|_{H} .
$$

If $\beta<0$, the estimate (2.21) remains valid (and results in hyperbolic sines of $|\beta|^{1 / 2} t$ ).

Remark. It is sufficient to prove (2.21). The case $\beta<0$ can be handled with minor modifications in the proof, such as replacing $\operatorname{Cos} \theta$ by $\operatorname{Cosh} \theta$, etc. The resulting hyperbolic sines imply an exponential decay of the data $f_{1}$ and $f_{2}$. This was used in [6] to construct an $A$-stable scheme. The estimate (2.22) follows from (2.21) on using L'Hospital's rule. It was obtained in [5] without the use of Chebyshev polynomials. The present discussion unifies and extends previous results. All of these results are quite plausible. Assume for simplicity that $g(t) \equiv 0$ in (2.3) and that $A(t) \equiv A$ is independent of $t$ and is selfadjoint with a discrete spectrum. Let the eigenvalues of $A$ satisfy

$$
\lambda(A) \geqq-\beta .
$$

Expanding in the eigenvectors of $A$, one can construct the exact solution of (2.3), (2.4). This yields the analog of $(2.21),(2.22)$ for the continuous problem. The reader who is convinced by this argument may proceed directly to Section 3 without fear of losing continuity.

To prove Theorem 1, we will show that $Q^{-1}(\Delta t)$ exists and is a bounded operator on all of $H^{N}$. As in [5] and [6], this can be done via the technique of "block Gaussian elimination" applied to the tridiagonal matrix $Q$. We will need the following lemmas.

LEMMA 2. The Chebyshev polynomials of the second kind on $[-1,1]$ can be generated by means of the recurrence relation 


$$
U_{n+1}(x)=2 x U_{n}(x)-U_{n-1}(x), \quad n \geqq 1,
$$

where $U_{0}(x)=1, U_{1}(x)=2 x$.

Putting $x=\operatorname{Cos} \theta$, we have

$$
U_{n}(x)=\operatorname{Sin}(n+1) \theta / \operatorname{Sin} \theta .
$$

Proof. See for example [20, p. 297].

LEMMA 3. Fix $a \theta>0$ sufficiently small such that $(N+1) \theta<\pi$. Then

$$
0<\operatorname{Sin} k \theta / \operatorname{Sin}(k+1) \theta<2 \operatorname{Cos} \theta
$$

for each $k=1,2, \cdots, N$.

Proof. The inequality is true for $\theta=0$. Hence, it remains true in a neighborhood of $\theta=0$.

LEMMA 4. Let $\pi^{2} / T^{2}>\beta$. Let $\Delta t=T /(N+1)$ be sufficiently small, and put $x=1-\beta \Delta t^{2} / 2$. Let $\Lambda_{1}=\left[2 / \Delta t^{2}+A^{1}\right]$ and let $\Gamma_{1}=-\left(\Lambda_{1}\right)^{-1}$. For each $n=2$, $3, \cdots, N$, let $\Lambda_{n}$ be given by

$$
\Lambda_{n}=\left[2 / \Delta t^{2}+A^{n}\right]+\Gamma_{n-1} / \Delta t^{4},
$$

where

$$
\Gamma_{n}=-\left(\Lambda_{n}\right)^{-1}, \quad n=2,3, \cdots, N .
$$

Then, for each $n=1,2, \cdots, N, \Gamma_{n}$ exists and is a bounded operator on $H$. Moreover,

$$
\left\|\Gamma_{n}\right\|_{H} / \Delta t^{2} \leqq U_{n-1}(x) / U_{n}(x), \quad n=1,2, \cdots, N,
$$

where $U_{n}$ is the nth Chebyshev polynomial of the second kind.

Proof. From (2.1), we have that, for each $n,\left[2 / \Delta t^{2}+A^{n}\right]$ is a closed invertible operator whose inverse has domain $H$ and

$$
\left\|\left(\frac{2}{\Delta t^{2}}+A^{n}\right)^{-1}\right\|_{H} \leqq \frac{\Delta t^{2}}{2-\beta \Delta t^{2}}=\frac{\Delta t^{2}}{2 x} .
$$

Hence,

$$
\left\|\Gamma_{1}\right\|_{H} / \Delta t^{2} \leqq 1 / 2 x=U_{0}(x) / U_{1}(x) .
$$

Suppose now that, for some positive integer $k<N, \Gamma_{k}$ exists and is a bounded operator with domain $H$, and

$$
\left\|\Gamma_{k}\right\|_{H} / \Delta t^{2} \leqq U_{k-1}(x) / U_{k}(x)
$$

In that case, we have

$$
\frac{1}{\Delta t^{4}}\left\|\Gamma_{k}\right\|_{H}\left\|\left(\frac{2}{\Delta t^{2}}+A^{k+1}\right)^{-1}\right\|_{H} \leqq \frac{U_{k-1}(x)}{2 x U_{k}(x)} .
$$

Putting $\operatorname{Cos} \theta=x=1-\beta \Delta t^{2} / 2$, we see that $\theta=\beta^{1 / 2} \Delta t+O\left(\Delta t^{2}\right)$. Hence, if $\Delta t$ is small enough, $\theta$ satisfies the hypotheses of Lemma 3. Therefore,

$$
U_{k-1}(x) / 2 x U_{k}(x)<1 .
$$

We may now employ the well-known lemma on the stability of bounded invertibility $\left[13\right.$, p. 196] to conclude that $\Gamma_{k+1}$ exists and is a bounded operator on $H$ and that 


$$
\frac{1}{\Delta t^{2}}\left\|\Gamma_{k+1}\right\|_{H} \leqq \frac{1 / 2 x}{1-U_{k-1}(x) / 2 x U_{k}(x)} .
$$

Using the recursion formula in Lemma 2, we get, from (2.35),

$$
\left\|\Gamma_{k+1}\right\|_{H} / \Delta t^{2} \leqq U_{k}(x) / U_{k+1}(x) .
$$

Hence, in view of (2.31), the lemma follows by induction.

Lemma 5. Let $Q$ be the matrix in (2.19). Let $\Lambda_{k}, \Gamma_{k}$ be as in Lemma 4. Let $Z=\left\{z^{k}\right\}$ be a given vector in $H^{N}$. Then, $X=\left\{x^{k}\right\}$ is a solution of $Q X=Z$ if and only if

$$
\begin{aligned}
x^{N} & =w^{N}, \\
x^{N-1}+\Gamma_{N-1} x^{N} / \Delta t^{2} & =w^{N-1}, \\
\vdots & \vdots \\
x^{1}+\Gamma_{1} x^{2} / \Delta t^{2} & =w^{1},
\end{aligned}
$$

where the $\left\{w^{i}\right\}$ are defined by

$$
\begin{array}{cc}
\Lambda_{1} w^{1}=z^{1}, \\
\Lambda_{2} w^{2}=z^{2}+w^{1} / \Delta t^{2}, \\
\vdots \\
\Lambda_{N} w^{N}=z^{N}+w^{N-1} / \Delta t^{2} .
\end{array}
$$

Proof. See [5, Lemma 4]. Since $\Lambda_{n}^{-1}$ is defined on all of $H$, Lemma 5 actually proves the existence of a unique solution of $Q X=Z$ for any $Z \in H^{N}$.

Lemma 6. Let $\pi^{2} / T^{2}>\beta$, and let $\Delta t=T /(N+1)$ be sufficiently small. Let $F \in H^{N}$ be defined as in (2.17) and let $Y=Q^{-1} F$. Then, for each $n=1,2, \cdots, N$, we have

$$
\begin{aligned}
\left\|y^{n}\right\|_{H} \leqq & \frac{\operatorname{Sin} \beta^{1 / 2}(T-n \Delta t)}{\operatorname{Sin} \beta^{1 / 2} T}\left\|f_{1}\right\|_{H} \\
& +\frac{\operatorname{Sin} \beta^{1 / 2} n \Delta t}{\operatorname{Sin} \beta^{1 / 2} T}\left\|f_{2}\right\|_{H}+O(\Delta t)\left\{\left\|f_{1}\right\|_{H}+\left\|f_{2}\right\|_{H}\right\}
\end{aligned}
$$

as $\Delta t \downarrow 0$.

Proof. Write $F=F^{1}+F^{2}$ where $F^{1}$ is $F$ with $f_{2}=0$ and $F^{2}$ is $F$ with $f_{1}=0$. Then $Y=Q^{-1} F^{1}+Q^{-1} F^{2}$. Consider first the contribution $Q^{-1} F^{2}$. Using Lemma 5 with $z^{1}=z^{2}=\cdots=z^{N-1}=0, z^{N}=f_{2} / \Delta t^{2}$, we get $w^{1}=w^{2}=\cdots=w^{N-1}=0$ and

$$
\left\|w^{N}\right\|_{H} \leqq \frac{U_{N-1}(x)}{U_{N}(x)}\left\|f_{2}\right\|_{H}
$$

on using the estimates in Lemma 4. Inserting this in Eqs. (2.37), we get

$$
\left\|y_{2}^{n}\right\|_{H} \leqq \frac{U_{n-1}(x)}{U_{N}(x)}|| f_{2} \|_{H}, \quad n=1,2, \cdots, N .
$$

In terms of $\theta=\operatorname{Arc} \operatorname{Cos} x$, we have, from Lemma 2, 


$$
\left\|y_{2}^{n}\right\|_{H} \leqq \frac{\operatorname{Sin} \beta^{1 / 2} n \Delta t}{\operatorname{Sin} \beta^{1 / 2} T}\left\|f_{2}\right\|_{H}+O(\Delta t)\left\|f_{2}\right\|_{H} .
$$

The estimate for the contribution $Q^{-1} F^{2}$ follows by symmetry.

We are now ready to prove Theorem 1 .

Proof of Theorem 1. By Lemma 5, $Q^{-1}$ exists and is defined on all of $H^{N}$. Since each $A^{n}$ is closed, $Q^{-1}$ is closed. Hence, $Q^{-1}$ is bounded on using the closed graph theorem. We proceed to derive the inequality (2.21). Let $M$ be the tridiagonal matrix given by

$$
M=Q-\operatorname{diag}\left\{A^{1}, A^{2}, \cdots, A^{N}\right\}
$$

Using the method of Lemma 6 in [5], one easily shows that, given any $\epsilon>0$, there exists $\Delta t$ sufficiently small so that

$$
\frac{(1-\epsilon) \pi^{2}}{T^{2}}(V, V) \leqq(M V, V)=\Delta t \sum_{k=0}^{N}\left\|v^{k+1}-v^{k}\right\|_{H}^{2} / \Delta t^{2},
$$

for every $V \in H^{N}$. In the above sum, $v^{0}, v^{N+1}$ are defined to be zero. If $W$ is in the domain of $Q$,

$$
(Q W, W)=(M W, W)+\Delta t \sum_{n=1}^{N}\left\langle A^{n} w^{n}, w^{n}\right\rangle .
$$

Hence, using (2.2) and (2.44),

$$
\operatorname{Re}(Q W, W) \geqq\left[(1-\epsilon) \pi^{2} / T^{2}-\beta\right](W, W) .
$$

Choosing $\epsilon$ small enough such that $(1-\epsilon) \pi^{2} / T^{2}>\beta$, we get, from (2.46),

$$
\left\|Q^{-1}\right\|_{H^{N}} \leqq \frac{T^{2}}{(1-\epsilon) \pi^{2}-\beta T^{2}} .
$$

Next, let $Q V=F-G$ with $F, G$ defined in (2.17), (2.18), and put

$$
W=V-Q^{-1} F \text {, }
$$

so that

$$
Q W=Q V-F=-G
$$

From (2.47), we get

$$
\|W\|_{H^{N}} \leqq \frac{T^{2}}{(1-\epsilon) \pi^{2}-\beta T^{2}}\|G\|_{H^{N}}
$$

Also, using Schwarz's inequality,

$$
\|W\|_{H^{N}}|| Q W\left\|_{H^{N}} \geqq|(Q W, W)| \geqq \operatorname{Re}(Q W, W) \geqq(M W, W)-\beta\right\| W \|_{H^{N}}^{2} .
$$

Using (2.49), (2.50), it follows from (2.51) that

$$
(M W, W) \leqq \frac{(1-\epsilon) \pi^{2} T^{2}}{\left[(1-\epsilon) \pi^{2}-\beta T^{2}\right]}\|G\|_{H^{N}}^{2}
$$

Now, for any $n$ with $1 \leqq n \leqq N$, we have

$$
w^{n}=\Delta t \sum_{k=0}^{n-1}\left(w^{k+1}-w^{k}\right) / \Delta t
$$


and, using Schwarz's inequality,

$$
\begin{aligned}
\left\|w^{n}\right\|_{H} & \leqq \Delta t \sum_{k=0}^{n-1} \frac{\left\|w^{k+1}-w^{k}\right\|_{H}}{\Delta t} \\
& \leqq\left\{\Delta t \sum_{k=0}^{N} 1\right\}^{1 / 2}\left\{\Delta t \sum_{k=0}^{N} \frac{\left\|w^{k+1}-w^{k}\right\|_{H}^{2}}{\Delta t^{2}}\right\}^{1 / 2} .
\end{aligned}
$$

Since

$$
(M W, W)=\Delta t \sum_{k=0}^{N}\left\|w^{k+1}-w^{k}\right\|_{H}^{2} / \Delta t^{2},
$$

we get from (2.54) and (2.52),

$$
\left\|w^{n}\right\|_{H} \leqq \frac{\pi T^{3 / 2}}{\left[(1-\epsilon) \pi^{2}-\beta T^{2}\right]}\|G\|_{H^{N}} .
$$

Finally, to estimate the components $\left\{v^{n}\right\}$ of the solution $V$ of $Q V=F-G$, recall the definition of $W$ in (2.48) and use the estimates of Lemma 6 for the components of $Q^{-1} F$. This yields the inequality (2.21) of Theorem 1 .

3. Linear Parabolic Problems and the Backward Beam Equation. To elucidate the connection between parabolic problems and the two-point problem of Section 2 , consider the following simple example. Let $R$ be the strip $\{(x, t) \mid 0 \leqq x \leqq 1, t \geqq 0\}$ in the $(x, t)$-plane and consider the mixed problem for the one-dimensional heat equation

$$
\begin{aligned}
u_{t} & =u_{x x}, & & 0<x<1, t>0, \\
u(0, t) & =u(1, t)=0, & & t \geqq 0, \\
u(x, 0) & =f_{1}(x), & & 0 \leqq x \leqq 1,
\end{aligned}
$$

where $f_{1}(x)$ is such that the unique solution of (3.1)-(3.3) has sufficiently many derivatives, bounded on $\bar{R}$. If we differentiate (3.1) with respect to time, we get

$$
u_{t t}=u_{x x x x}, \quad 0<x<1 \text {. }
$$

Since $u$ is zero on the lateral sides of the strip $R$, it follows from (3.1) that $u_{x x}$ is also zero there. Suppose that one knows the exact solution of (3.1)-(3.3) at some positive time $T$. Let $u(x, T)=f_{2}(x)$. Then, the unique solution of (3.1)-(3.3) satisfies the following auxiliary system:

$$
\begin{aligned}
v_{t t} & =v_{x x x x}, \quad 0<x<1,0<t<T, \\
v(0, t) & =v_{x x}(0, t)=v_{x x}(1, t)=v(1, t)=0, \quad t \geqq 0, \\
v(x, 0) & =f_{1}(x), \\
v(x, T) & =f_{2}(x) .
\end{aligned}
$$

One may write the above system in the more compact form of a differential equation in the Hilbert space $L^{2}[0,1]$,

$$
\begin{array}{rlrl}
v_{t t}-A v & =0, & 0<t<T, \\
v(0) & =f_{1}, & & v(T)=f_{2},
\end{array}
$$


where now $v(t) \equiv v(\cdot, t)$ is a Hilbert space valued function on $[0, T]$, and $A$ is the unbounded operator in $L^{2}[0,1]$ corresponding to the spatial part of $(3.5)$ with the boundary conditions (3.6). Thus, $A$ is a positive selfadjoint operator in $L^{2}$; it is exactly the square of the operator defined by the spatial part of (3.1) with the boundary conditions (3.2). Hence, in this case,

$$
\langle A v, v\rangle \geqq \pi^{4}\langle v, v\rangle \quad \forall v \in D(A) .
$$

One may use the energy inequality of Lemma 1 , with $\beta=0$, to prove uniqueness in (3.5)-(3.8). Consequently, the solution of (3.1)-(3.3) can be obtained by solving instead the system (3.5)-(3.8). We call (3.5) the "backward beam equation" because of its similarity with the vibrating beam equation $v_{t t}=-v_{x x x x}$. In contrast to the latter, the initial-value problem is not well posed for the backward beam equation. What is well posed is the "initial-terminal" problem (3.10). This is easily seen by separation of variables which leads to the estimate

$$
\|v(t)\|_{2} \leqq \frac{\operatorname{Sinh} \pi^{2}(T-t)}{\operatorname{Sinh} \pi^{2} T}\left\|f_{1}\right\|_{2}+\frac{\operatorname{Sinh} \pi^{2} t}{\operatorname{Sinh} \pi^{2} T}\left\|f_{2}\right\|_{2} .
$$

Recall that $f_{2}$ in (3.8) is the exact solution of the heat conduction problem at time $T$. Hence, $f_{2}$ is necessarily an analytic function. Imagine now solving (3.5)-(3.8) with $f_{2}$ replaced by $\tilde{f}_{2}$, a function close to $f_{2}$ in the $L^{2}$ norm, but not necessarily analytic. In that case, the corresponding solution of the backward beam equation cannot in general be a solution of the heat conduction problem. However, because of (3.12), this solution is close in the $L^{2}$ norm to the solution of the heat equation determined by $f_{1}(x)$.

One can relate more general linear parabolic problems to the two-point problem of Section 2. Consider a parabolic equation, in some bounded domain $\Omega$ in $R^{n}$,

$$
u_{t}=-P_{0}(t) u, \quad 0<t \leqq T,
$$

together with certain homogeneous conditions, on the smooth boundary of $\Omega$,

$$
B[u]=0 \quad \text { on } \partial \Omega .
$$

Here, $P_{0}(t)$ is a uniformly elliptic operator in the space variables with coefficients depending smoothly on $x$ and $t$. Let $\dot{P}_{0}(t)$ be the differential operator obtained from $P_{0}(t)$ by differentiating the coefficients with respect to $t$. We then have that any smooth solution of (3.13) satisfies

$$
u_{t t}=\left[P_{0}^{2}-\dot{P}_{0}\right] u
$$

and

$$
B[u]=0 \text { on } \partial \Omega .
$$

However, since $P_{0}^{2}$ is of higher order than $P_{0}$, one must find extra boundary conditions, also satisfied by a smooth solution of (3.13), in order to obtain this solution uniquely from (3.15). Suppose that the boundary conditions (3.16) are independent of $t$. Then we may differentiate both sides of (3.16) with respect to $t$ to obtain

$$
B\left[u_{t}\right]=0 \text { on } \partial \Omega .
$$

Using (3.13), this leads to the auxiliary problem 


$$
\begin{aligned}
u_{t} & =\left[P_{0}^{2}-\dot{P}_{0}\right] u, & & x \in \Omega, 0<t \leqq T, \\
B[u] & =B\left[P_{0} u\right]=0 & & \text { on } \partial \Omega, 0<t \leqq T .
\end{aligned}
$$

In [6, Section 3], an analysis is carried out on the auxiliary problem (3.18), (3.19) generated by a class of selfadjoint problems which are $V$-parabolic in the sense of Lions [14], [19]. The elliptic operator $P_{0}(t)$ in (3.13) together with the boundary conditions (3.14) are assumed defined via a symmetric bilinear form

$$
a(t ; u, v)=\sum_{|p|,|q| \leq m} \int_{\Omega} a_{p Q}(x, t) D^{a} u \overline{D^{p} v} d x,
$$

which is continuous and strongly coercive on a Hilbert space $V$, lying between the Sobolev spaces $H_{0}^{m}(\Omega)$ and $H^{m}(\Omega)$. Using the Lax-Milgram lemma, the following result is proved [6, Theorem 1]:

THEOREM 2. Consider the parabolic equation

$$
u_{t}=-P(t) u, \quad 0<t<T,
$$

where, for each $t, P(t)$ is the positive selfadjoint operator in $L^{2}(\Omega)$ defined by the strongly coercive bilinear form (3.20). Let the domain of $P(t)$ in $L^{2}(\Omega)$ be independent of $t$, and let $u(t)$ be a sufficiently smooth solution of (3.21). Then $u(t)$ also satisfies the equation

$$
u_{t t}-A(t) u=0, \quad 0<t<T,
$$

where $A(t)$ is the unbounded operator in $L^{2}(\Omega)$, defined by the right-hand side of (3.18) and the boundary conditions (3.19). Moreover, there exists a constant $\beta \geqq 0$ and independent of $t$, such that $A(t)+\beta$ is an m-accretive operator in $L^{2}(\Omega)$.

Remark. In fact, $A(t)$ is selfadjoint in Theorem 2; the need for an additive constant $\beta$ to render $A(t)$ accretive is due to the extra term $\dot{P}_{0}(t)$ which may spoil the positivity of $P^{2}$. This extra term is absent in the time independent case. Note that the domain of $A(t)$ may vary with $t$ even though $P(t)$ is assumed to have fixed domain. See [6, Section 3].

Consider now the question of approximating the solution of (3.21) by considering instead (3.22). By assumption, $u$ is sufficiently smooth so that Lemma 1 applies. Suppose $T$ is such that $\beta^{1 / 2} T<\pi$ and let $f_{2}(x)$ be the exact solution of (3.21) at time $T$. The difference scheme (2.10), (2.11) now provides a stable "method of lines" where only the time variable is discretized while the space variables remain continuous. In an actual calculation, the spatial operator must also be approximated. In order to maintain the stability inequalities for the fully-discrete scheme, we see from Theorem 1 that it is sufficient to do the space discretization in such a way that the discrete analog of (2.2) remains valid, uniformly in the spatial mesh size $\Delta x$ as $\Delta x \rightarrow 0$. In the remainder of this section, we show how to do this, using finite-differences, for a class of two-dimensional second-order problems. More general problems will be considered in a later report using Galerkin methods.

3.1. The Fully Discrete Scheme for $u_{t}=\nabla \cdot a \nabla u-c u$ on a Rectangle. Let $\Omega$ be a rectangle in the $(x, y)$-plane and consider the parabolic problem

$$
\begin{gathered}
u_{t}=\left[a(x, y, t) u_{x}\right]_{x}+\left[a(x, y, t) u_{y}\right]_{y}-c(x, y, t) u, \quad(x, y) \in \Omega, t>0, \\
u(x, y, 0)=f_{1}(x, y), \quad x, y \in \Omega, \\
u(x, y, t)=0, \quad(x, y) \in \partial \Omega, t \geqq 0 .
\end{gathered}
$$


We assume $a, c$ and $f$ to be smooth functions with

$$
a(x, y, t) \geqq a_{0}>0, \quad c(x, y, t) \geqq 0 .
$$

Let $-P_{0}(t)$ denote the elliptic operator on the right of (3.23) at time $t$. Introduce a rectangular mesh region $\Omega(\Delta x)$ over $\Omega$ with mesh spacing $\Delta x=\Delta y$, and $J$ interior mesh points. At each $t=n \Delta t$, we approximate $P_{0}(t)$ by the usual centered five-point difference analog

$$
\begin{array}{rlrl}
-\left(P^{n}(\Delta x) v^{n}\right)(x, y) & =\left[a^{n}\left(x+\frac{\Delta x}{2}, y\right) v_{x}^{n}\right]_{\bar{x}}+\left[a^{n}\left(x, y+\frac{\Delta y}{2}\right) v_{\nu}^{n}\right]_{\nu}-c^{n} v^{n}, \\
v^{n}(x, y) & =0, \quad(x, y) \in \partial \Omega(\Delta x), & (x, y) \in \Omega(\Delta x),
\end{array}
$$

where $v_{x}^{n}, v_{x}^{n}$ denote the forward and backward difference quotients of $v^{n}(x, y)$ over $\Omega(\Delta x)$.

Denote by $V^{n}$ the $J$-component vector formed from $v^{n}(x, y)$ at the interior mesh points. Let $P^{n}(\Delta x)$ denote the $J \times J$ real symmetric matrix corresponding to the difference operator (3.27) and the boundary conditions (3.28). We use the notation

$$
\begin{aligned}
\left\langle V^{n}, W^{n}\right\rangle & =(\Delta x)^{2} \sum_{(x, y) \in \Omega(\Delta x)} V^{n}(x, y) \overline{W^{n}(x, y)}, \\
\left\|V^{n}\right\|_{2}^{2} & =\left\langle V^{n}, V^{n}\right\rangle,
\end{aligned}
$$

for discrete scalar products and norms of $J$-vectors.

Let $L(\Delta x)$ be the $J \times J$ matrix corresponding to the five-point difference analog of the negative Laplacian with the boundary conditions (3.28). Then, using (3.26) and summation by parts, we have

$$
a_{0}\left\langle L W^{n}, W^{n}\right\rangle \leqq\left\langle P^{n} W^{n}, W^{n}\right\rangle \leqq a_{1}\left\langle L W^{n}, W^{n}\right\rangle+c_{1}\left\|W^{n}\right\|_{2}^{2},
$$

for all $J$-component vectors $W^{n}$. Here $a_{1}$ and $c_{1}$ are, respectively, upper bounds for $a(x, y, t)$ and $c(x, y, t)$. Since $L$ is positive definite independently of $\Delta x$ as $\Delta x \rightarrow 0$, we see that $P^{n}$ is positive definite, uniformly in $n$ and in $\Delta x$ as $\Delta x \rightarrow 0$. Let

$$
\partial a / \partial t \leqq \alpha, \quad \partial c / \partial t \leqq \gamma .
$$

Define $\dot{P}^{n}$ to be the symmetric matrix obtained from $P^{n}$ by replacing $a(x, y, t)$ and $c(x, y, t)$, respectively, by $\partial a / \partial t, \partial c / \partial t$. From (3.32), we get

$$
\left\langle\dot{P}^{n} W^{n}, W^{n}\right\rangle \leqq \alpha\left\langle L W^{n}, W^{n}\right\rangle+\gamma\left\|W^{n}\right\|_{2}^{2} .
$$

Next, let $f_{2}(x, y)$ be the exact solution of (3.23)-(3.25) at time $T$. If we now differentiate with respect to time in (3.23), we get the auxiliary problem

$$
\begin{aligned}
u_{\imath t} & =\left[P_{0}^{2}(t)-\dot{P}_{0}(t)\right] u, & & (x, y) \in \Omega, 0<t<T, \\
u(x, y, 0) & =f_{1}(x, y), & & u(x, y, T)=f_{2}(x, y), \\
u & =P_{0}(t) u=0 & & \text { on } \partial \Omega, 0 \leqq t \leqq T .
\end{aligned}
$$

This is the problem to be discretized. Define the $J \times J$ matrix $A^{n}(\Delta x)$ by

$$
A^{n}(\Delta x)=\left(P^{n}\right)^{2}-\dot{P}^{n} .
$$


A little thought shows that $A^{n}(\Delta x)$ is an $O\left(\Delta x^{2}\right)$ difference approximation at $t=n \Delta t$ to the spatial operator on the right of (3.34) together with the boundary conditions (3.36). Hence, the fully-discrete scheme for the auxiliary problem (3.34)-(3.36) is obtained by replacing $u_{t}$ in (3.34) by a centered second-difference quotient, while using (3.37) for the spatial operator. Using the natural ordering along lines $t=$ constant, the fully discrete scheme can be written as

$$
Q(\Delta x) V=F,
$$

where $Q(\Delta x)$ is the matrix (2.19) with $A^{n}$ replaced by $A^{n}(\Delta x), V$ is the "block" vector

$$
V=\left\{V^{1}, V^{2}, \cdots, V^{N}\right\}^{T},
$$

and $F$ contains the data $f_{1}, f_{2}$ evaluated on $\Omega(\Delta x)$. We now establish the analog of (2.2) for $A^{n}(\Delta x)$. In the simple but important case where (3.32) holds with $\alpha=\gamma=0$, we see that $\dot{P}^{n}$ is negative semidefinite and hence $A^{n}(\Delta x)$ is positive definite. More generally,

$$
\begin{aligned}
\left|\left\langle\dot{P}^{n} W^{n}, W^{n}\right\rangle\right| & \leqq\left\|a_{t}\right\|_{\infty}\left\langle L W^{n}, W^{n}\right\rangle+\left\|c_{t}\right\|_{\infty}\left\|W^{n}\right\|_{2}^{2} \\
& \leqq\left(\left\|a_{t}\right\|_{\infty}+\left\|c_{t}\right\|_{\infty} / \lambda_{0}\right)\left\langle L W^{n}, W^{n}\right\rangle,
\end{aligned}
$$

where $\lambda_{0}>0$ is the infimum of the eigenvalues of $L$ as $\Delta x \rightarrow 0$. Consequently, if

$$
\chi=\left\|a_{t}\right\|_{\infty} / a_{0}+\left\|c_{t}\right\|_{\infty} / a_{0} \lambda_{0}
$$

we have, from (3.31),

$$
\chi\left\langle P^{n} W^{n}, W^{n}\right\rangle-\left\langle\dot{P}^{n} W^{n}, W^{n}\right\rangle \geqq 0 .
$$

Therefore, from (3.37) and (3.42),

$$
\left\langle A^{n} W^{n}, W^{n}\right\rangle \geqq\left\|P^{n} W^{n}\right\|_{2}^{2}-\chi\left\langle P^{n} W^{n}, W^{n}\right\rangle .
$$

Using the inequality

$$
\epsilon^{2}\left\|P^{n} W^{n}\right\|-\chi\left\langle P_{n} W_{n}, W_{n}\right\rangle+\chi^{2}\left\|W^{n}\right\|_{2}^{2} / 4 \epsilon^{2} \geqq 0,
$$

we obtain from (3.43), for every $0<\epsilon<1$,

$$
\left\langle A^{n} W^{n}, W^{n}\right\rangle \geqq\left(1-\epsilon^{2}\right)\left\|P^{n} W^{n}\right\|_{2}^{2}-\chi^{2}\left\|W^{n}\right\|_{2}^{2} / 4 \epsilon^{2} .
$$

Hence, using (3.31),

$$
\left\langle A^{n} W^{n}, W^{n}\right\rangle \geqq\left[a_{0} \lambda_{0}^{2}\left(1-\epsilon^{2}\right)-\chi^{2} / 4 \epsilon^{2}\right]\left\|W^{n}\right\|_{2}^{2} .
$$

This proves (2.2) for $A^{n}(\Delta x)$ with a constant $\beta$ depending on $\left\|a_{t}\right\|_{\infty}$ and $\left\|c_{t}\right\|_{\infty}$. Note that $\beta$ is positive if $\left\|a_{t}\right\|_{\infty}$ and $\left\|c_{t}\right\|_{\infty}$ are sufficiently large. From Theorem 1 , we see that this leads to a restriction on $T$. The reason for this restriction will be seen in the next section, where it will be eliminated by suitably transforming the original problem (3.23).

A discussion of methods for solving the difference equations (3.38) will appear in another report. In the case of one space dimension, one may use the block Gaussian elimination scheme discussed in the proof of Theorem 1. Other methods include SLOR, S2LOR, and in the case of time-independent coefficients, the matrix decomposition method of [2]. The latter method is the one used in the example of Section 5. 
4. Parabolic Equations Backwards in Time. Consider the parabolic equation

$$
u_{t}=-P(t) u, \quad t>0,
$$

where, for each $t, P(t)$ is a positive selfadjoint operator arising from a coercive elliptic boundary-value problem, as is the case in Theorem 2 of Section 3. The backward problem associated with (4.1) is, given a function $f_{2}(x)$ in $L^{2}(\Omega)$, to find a solution of (4.1) which at time $T>0$ takes on the value $f_{2}$. As is well known, there cannot, in general, exist a solution, unless $f_{2}$ meets certain smoothness requirements. On the other hand, if there is a solution, it is unique. For results on backward uniqueness, see [17], [18] and [11]. In practice, one cannot measure $f_{2}$ with sufficient precision to determine whether or not $f_{2}$ meets the necessary requirements for existence. However, in a real problem, one will know that $f_{2}$ is close, in the $L^{2}$ norm, to a function $\tilde{f}_{2}$ for which a solution exists. Moreover, from physical considerations, one will know a bound on the solution at previous times. This bound need not be sharp. Hence, the following version of the backward problem makes sense. Given the positive constants $\delta, M, T$, and given $f_{2}(x)$ in $L^{2}(\Omega)$, find all solutions of $(4.1)$ on $[0, T]$ which satisfy

$$
\begin{aligned}
\|u(0)\|_{2} & \leqq M, \\
\left\|u(T)-f_{2}\right\|_{2} & \leqq \delta .
\end{aligned}
$$

This is the version of the problem that is considered in this paper. It is clear that if there is one solution to this problem, there are, in general, infinitely many. Our method will produce a mesh function which is an approximation to all of them simultaneously. Concerning the question of continuous dependence and error bounds in the above problem, the following results are known. First, assume $P(t)$ in $(4.1)$ to be independent of $t$. Let $u(t)$ be a solution of (4.1). Then (see [11, p. 183]), $\log \|u(t)\|_{2}$ is a convex function of $t$ and, therefore, for $0 \leqq t \leqq T$,

$$
\|u(t)\|_{2} \leqq\|u(0)\|_{2}^{(T-t) / T}\|u(T)\|_{2}^{t / T} .
$$

Hence, if $u_{1}(t)$ and $u_{2}(t)$ are any two solutions of (4.1) on [0,T] satisfying (4.2), (4.3), and if $\epsilon(t)$ is their difference, we have the following stability estimate:

$$
\|\epsilon(t)\|_{2} \leqq(2 M)^{(T-t) / T}(2 \delta)^{t / T}, \quad 0 \leqq t \leqq T .
$$

In the case where $P(t)$ in (4.1) depends on $t$, a less generous convexity result, due to Agmon and Nirenberg, is known. (See, e.g., [11, p. 182].) Specifically, there exist positive constants $m$ and $c$ so that if $\mu=\mu(t)$ is given by

$$
\mu(t)=\left(e^{c T}-1\right) /\left(e^{c t}-1\right),
$$

then any smooth solution of (4.1) satisfies

$$
\|u(t)\|_{2} \leqq e^{m t} e^{-m T / \mu}\|u(0)\|_{2}^{(\mu-1) / \mu}\|u(T)\|_{2}^{1 / \mu}, \quad 0 \leqq t \leqq T .
$$

Suppose for simplicity that one can take $m=0$ in (4.7) and consider the resulting inequality at $t=T / 2$. Using

$$
\mu(T / 2) \approx e^{c T / 2},
$$

we get, from (4.7), the following result for the difference $\epsilon(t)$ of any two solutions satisfying (4.2), (4.3): 


$$
\|\epsilon(T / 2)\|_{2} \leqq(2 M)^{1-\mathrm{e}-\mathrm{c} T / 2}(2 \delta)^{-\mathrm{c} T / 2} .
$$

Suppose now that $c$ and $T$ are such that

$$
e^{-c T / 2} \ll \frac{1}{2} .
$$

A comparison of (4.9) with (4.5) at $t=T / 2$ suggests the following. In order to know the solution of the time-dependent problem with the same certainty as in the timeindependent case, one must, in general, know the corresponding terminal data with much greater accuracy. This seems plausible on the following grounds. Consider the simple parabolic problem

$$
\begin{aligned}
u_{t} & =\left[a(x, t) u_{x}\right]_{x}, & & 0<x<1, t>0, \\
u(x, 0) & =f_{1}(x), & & 0 \leqq x \leqq 1, \\
u(0, t) & =u(1, t)=0, & & t \geqq 0 .
\end{aligned}
$$

If the diffusion coefficient $a(x, t)$ in (4.11) is an increasing function of time, it is evident that the initial data $f_{1}(x)$ is smoothed out at a higher rate than is the case when $\partial a / \partial t \leqq 0$. Consequently, more precision in measurement is necessary at time $T$, in order to obtain the same amount of information as in the case when $\partial a / \partial t \leqq 0$.

We have gone into the above discussion in order to set the stage for our method. We begin with the simpler case of $P$ independent of $t$.

4.1. Selfadjoint Problems with Time-Independent Coefficients. Let $P$ be a positive selfadjoint operator in $L^{2}(\Omega)$ and let

$$
u_{t}=-P u, \quad 0<t<T .
$$

Let $f_{2}(x)$ be the given approximation to the terminal data at time $T$. The idea behind our method is to solve the backward problem for (4.14) by considering the backward beam equation, associated with (4.14),

$$
w_{t \iota}=P^{2} w, \quad 0<t<T,
$$

together with the initial-terminal conditions

$$
w(T)=f_{2}, \quad w(0)=0 .
$$

Note that the unknown initial data for (4.14) has been replaced by zero in the auxiliary problem. Consider now the error committed by using the wrong initial data. In analyzing this question, we may as well make a direct attack on the difference scheme which will be used to solve the auxiliary problem. Moreover, it is convenient and sufficient to consider only the "method of lines" of Section 2. All statements below will also hold for the fully discrete scheme of Section 3, provided continuous $L^{2}$ norms are replaced by discrete $L^{2}$ norms and the truncation error $O\left(\Delta t^{2}\right)$ is replaced by $O\left(\Delta t^{2}+\Delta x^{2}\right)$. Thus, we use the scheme (2.10), (2.11), with $f_{1}=0$ and $g^{n}=0$ to approximate (4.15), (4.16). Let $\epsilon(n \Delta t)$ be the difference between the solution of the difference equations and any of the exact solutions of (4.14) satisyfing (4.2), (4.3). We see that $\left\{\epsilon^{n}\right\}$ satisfies the difference equations (2.10), (2.11), with $g^{n}$ representing the truncation error, $f_{1}$ the initial data, and $f_{2}$ the difference between the exact and approximate terminal data. Since $P^{2} \geqq 0$, the estimate (2.22) is valid. Hence, using the given bounds (4.2), (4.3), we get, from (2.22), 


$$
\|\epsilon(t)\|_{2} \leqq M((T-t) / T)+\delta(t / T)+O\left(\Delta t^{2}\right), \quad 0 \leqq t \leqq T .
$$

A little thought shows that the error bound (4.17) is not satisfactory. Indeed, the error due to using the wrong initial data decays linearly with time, while the solutions of (4.14) decay exponentially with increasing time. Thus, eventually, a time will be reached where the error is bigger than the solution itself. On the other hand, (4.17) would be a useful bound if somehow the solutions of (4.14) grew exponentially sufficiently fast, for then the error due to the wrong initial data would eventually be imperceptible.

Thus, rather than deal with (4.14) directly, we set

$$
v=e^{k t} u, \quad 0 \leqq t \leqq T,
$$

in (4.14), where $k$ is a large positive integer chosen so that $v$ grows exponentially with time sufficiently fast. Then, $v$ satisfies

$$
v_{t}=-(P-k) v, \quad 0<t<T,
$$

and hence the auxiliary problem for $v$ is

$$
\begin{aligned}
w_{t \iota} & =(P-k)^{2} w, & & 0<t<T, \\
w(T) & =e^{k T} f_{2}, & & w(0)=0 .
\end{aligned}
$$

The essence of our method is to solve (4.20), (4.21) numerically in order to obtain a good approximation for $v$ and hence as good an approximation for $u$ in the original problem. Notice that since $P$ is selfadjoint, $(P-k)^{2}$ is selfadjoint nonnegative, and so the estimate (2.22) is still valid. Thus, if we use the scheme (2.10), (2.11) (with $f_{1}=0, g^{n}=0$, and $f_{2}$ replaced by $e^{k T} f_{2}$ ) to approximate (4.20), (4.21), and if $w_{\mathrm{app}}(t)$ is the solution of the difference equations, we find

$$
\left\|v(t)-w_{\mathrm{app}}(t)\right\|_{2} \leqq M((T-t) / T)+(t / T) e^{k T} \delta+O\left(\Delta t^{2}\right),
$$

for the difference between $w_{\mathrm{app}}(t)$ and any of the exact solutions of the modified backward problem (4.19). Let $\sigma(t)$ be the relative error in the $L_{2}$ norm, that is,

$$
\sigma(t)=\left\|v(t)-w_{\text {app }}(t)\right\|_{2} /\|v(t)\|_{2} .
$$

If $k$ is chosen properly in (4.18) and $\Delta t$ is sufficiently small, $\sigma(t)$ will be acceptably small on some interval $0<t_{0} \leqq t \leqq T$. In fact, if the terminal data is known with infinite precision, then, by choosing $k$ sufficiently large and $\Delta t$ sufficiently small, one can make $\sigma(t)$ acceptably small on as large a subinterval of $(0, T]$ as desired. Furthermore, since

$$
\sigma(t)=\frac{e^{-k t}\left\|v(t)-w_{\mathrm{app}}(t)\right\|_{2}}{e^{-k t}\|v(t)\|_{2}}=\frac{\left\|u(t)-e^{-k t} w_{\mathrm{app}}(t)\right\|_{2}}{\|u(t)\|_{2}},
$$

we see that

$$
u_{\mathrm{app}}(t)=e^{-k t} w_{\mathrm{apD}}(t)
$$

will be an equally good approximation to the solutions of the original backward problem for (4.14).

The fact that the terminal data $\tilde{f}_{2}(x)$ is only known to within $\delta$ results in an optimal choice for $k$, as was observed by P. D. Lax. From (4.22), we have 


$$
\left\|u(t)-e^{-k t} w_{\mathrm{app}}(t)\right\| \leqq M e^{-k t} \frac{(T-t)}{T}+\left(\frac{t}{T}\right) e^{k(T-t)} \delta+O\left(\Delta t^{2}\right) .
$$

Neglecting the term $O\left(\Delta t^{2}\right)$ in (4.26), we find that the right-hand side is minimized as a function of $k$ by choosing

$$
k_{0}=(1 / T) \log (M / \delta) .
$$

Moreover, with this choice of $k,(4.26)$ becomes

$$
\left\|u(t)-e^{-k_{0} t} w_{\mathrm{app}}(t)\right\|_{2} \leqq(M)^{(T-t) / T}(\delta)^{t / T}+O\left(\Delta t^{2}\right), \quad 0 \leqq t \leqq T .
$$

It is interesting to note that, apart from the truncation error, the bound (4.28) is the same as that in (4.5), which followed from the basic convexity result in the timeindependent case. It is fair to warn the reader that the truncation error term $O\left(\Delta t^{2}\right)$ contains a factor $\left(k_{0}\right)^{4}$ due to replacing $v_{t}$ by a second-difference quotient. Hence, in practice, the contribution from truncation will be an appreciable part of the total error in this method. For the fully discrete scheme of Section 3, the truncation error term on the right of (4.28) becomes $O\left[\left(k_{0}\right)^{4} \Delta t^{2}\right]+O\left(\Delta x^{2}\right)$; that is, the factor $\left(k_{0}\right)^{4}$ does not affect the error due to discretizing the space variables. In practice, this means that one will often choose $\Delta t$ much smaller than $\Delta x$ in order to minimize the truncation error.

4.2. Selfadjoint Problems with Time-Dependent Coefficients. The time-dependent case is more subtle. To develop the reader's insight, it is worthwhile to begin with the simple one-dimensional problem

$$
\begin{aligned}
u_{t} & =\left[a(x, t) u_{x}\right]_{x}-c(x, t) u, & & 0<x<1, t>0, \\
u(0, t) & =u(1, t)=0, & & t \geqq 0, \\
u(x, 0) & =f_{1}(x), & & 0 \leqq x \leqq 1,
\end{aligned}
$$

where the coefficients $a(x, t), c(x, t)$ are smooth functions with sufficiently many bounded derivatives and

$$
a(x, t) \geqq a_{0}>0, \quad c(x, t) \geqq 0 .
$$

In connection with the backward problem for (4.29), we distinguish three cases.

$$
\begin{aligned}
& \text { Case 1. } \frac{\partial a}{\partial t} \leqq 0, \quad \frac{\partial c}{\partial t} \leqq 0 . \\
& \text { Case 2. } \frac{\partial a}{\partial t} \leqq 0, \quad \frac{\partial c}{\partial t} \leqq \gamma, \quad \gamma>0 . \\
& \text { Case 3. } \frac{\partial a}{\partial t} \leqq \alpha, \quad \frac{\partial c}{\partial t} \leqq \gamma, \quad \alpha>0, \gamma \geqq 0 .
\end{aligned}
$$

In Case 1 , the diffusion coefficient $a(x, t)$ and the dissipative term $c(x, t)$ do not increase as $t$ increases. Hence, the smoothing undergone by the initial data $f_{1}(x)$ is no worse than would take place in the time-independent problem

$$
u_{t}=\left[a(x, 0) u_{x}\right]_{x}-c(x, 0) u .
$$

In Case 2, there is additional smoothing due to the growing dissipative term. In 
Case 3, the smoothing is much more severe due to the growing diffusion term. The qualitative difference between these three cases is borne out by the error bounds in our difference scheme.

Let us write the initial-boundary problem (4.29)-(4.31) in the form

$$
\begin{aligned}
u_{t} & =-Q(t) u, \quad t>0, \\
u(0) & =f_{1},
\end{aligned}
$$

where $Q(t)$ is the positive selfadjoint operator in $L^{2}[0,1]$ defined by

$$
Q_{0}(t) w=-\left[a(x, t) w_{x}\right]_{x}+c(x, t) w, \quad 0<x<1,
$$

and the boundary conditions

$$
w(0)=w(1)=0 .
$$

As in Section 3, we use the notation $Q_{0}(t)$ to denote the formal differential operator in (4.37) without the boundary conditions. By $\dot{Q}_{0}(t)$, we mean, as usual, the formal differential operator obtained from $Q_{0}(t)$ by differentiating its coefficients with respect to $t$. Thus, if we differentiate (4.35) with respect to $t$, we obtain the backward beam equation

$$
u_{t t}=B(t) u,
$$

where $B(t)$ is the unbounded operator in $L^{2}[0,1]$ defined by the formal differential operator

$$
B_{0}(t)=Q_{0}^{2}-\dot{Q}_{0}
$$

together with the boundary conditions

$$
w(0)=\left.Q_{0}(t) w\right|_{x=0}=w(1)=\left.Q_{0}(t) w\right|_{x=1}=0 .
$$

In dealing with the backward problem for (4.35), it will be necessary, as before, to consider the modified auxiliary problem obtained by setting

$$
v=e^{k t} u
$$

in (4.35), for a suitable positive $k$. This modified problem is

$$
v_{t t}=A(t) v,
$$

where $A(t)$ is the operator in $L^{2}$ defined by

$$
A_{0}(t)=\left(Q_{0}-k\right)^{2}-\dot{Q}_{0}
$$

together with the boundary conditions (4.41). We now observe the following. If $\dot{Q}(t)$ is the operator defined by $\dot{Q}_{0}(t)$ on the domain of $A(t)$ in (4.43), then

$$
\begin{array}{lll}
\langle\dot{Q}(t) v, v\rangle \leqq 0 & \forall v \in D_{A}, & \text { in Case 1, } \\
\langle\dot{Q}(t) v, v\rangle \leqq \gamma\langle v, v\rangle & \forall v \in D_{A}, & \text { in Case 2. }
\end{array}
$$

In Case 3, because of the growing diffusion coefficient, $\dot{Q}(t)$ is not semibounded from above in the $L^{2}$ norm. In Cases 1 and 2, it follows from (4.45), (4.46) that, for any $k$, we have, in (4.43), 


$$
\begin{array}{ll}
\langle A(t) v, v\rangle \geqq 0 \quad \forall v \in D_{A}, & \text { in Case 1, } \\
\langle A(t) v, v\rangle \geqq-\gamma\langle v, v\rangle, & \text { in Case 2. }
\end{array}
$$

In Case 3, a little thought reveals that $A(t)$ is semibounded from below, but the bound depends on $k$. This is not satisfactory for our purposes. We shall return to Case 3 later in this discussion.

Consider now the backward problem in Case 1. Let $f_{2}(x)$ be the given approximation to the terminal data at time $T$ for Eq. (4.35). By assumption, the solutions of (4.35) satisfy (4.2), (4.3). In (4.42) above, set $k=k_{0}$ where $k_{0}$ is defined in (4.27) and consider the auxiliary problem, for $v$,

$$
\begin{gathered}
w_{t t}-A(t) w=0, \quad 0<t<T, \\
w(T)=e^{k_{0} T} f_{2}, \quad w(0)=0 .
\end{gathered}
$$

Use the difference scheme (2.10), (2.11) to solve (4.49), (4.50). Since $A(t)$ satisfies (4.47), the estimate (2.22) is valid. Consequently, so is the estimate (4.28) applied to the present problem (4.35). Thus, Case 1 behaves like the time-independent problem (4.34).

In Case 2, more precise measurements are necessary at time $T$ in order to obtain the same amount of information about the solution as in Case 1. Hence, it may be anticipated that the estimate (4.28) cannot hold. An indication of the trouble is provided by Theorem 1 of Section 2 where we see that $T$ is restricted by the requirement $\gamma^{1 / 2} T<\pi$ and we lose uniqueness as $\gamma^{1 / 2} T \uparrow \pi$. To eliminate this difficulty, consider the preliminary transformation

$$
\tilde{u}=\exp \left\{\gamma t^{2}\right\} u
$$

in (4.29). Then $\tilde{u}$ satisfies

$$
\tilde{u}_{t}=\left[a(x, t) \tilde{u}_{x}\right]_{x}-[c(x, t)-\gamma t] \tilde{u} .
$$

Thus, (4.51) reduces Case 2 to Case 1. In fact, this transformation can be used for general abstract problems. It changes (4.35), (4.36) into the problem

$$
\begin{aligned}
\tilde{u}_{t} & =-\tilde{Q}(t) \tilde{u}_{0} \quad t>0, \\
\tilde{u}(0) & =f_{1},
\end{aligned}
$$

where $Q(t) \equiv Q(t)-\gamma t$, satisfies (4.45). Put

$$
\tilde{\delta}=\exp \left\{\gamma T^{2}\right\} \delta
$$

and let

$$
\tilde{k}_{0}=(1 / T) \log (M / \tilde{\delta}) .
$$

Note that with $k_{0}$ as in (4.27), we have

$$
\tilde{k}_{0}=k_{0}-\gamma T / 2 .
$$

We now solve the auxiliary problem

$$
w_{t t}-\tilde{A}(t) w=0, \quad 0<t<T,
$$




$$
w(T)=e^{\gamma T^{2} / 2} e^{\bar{k}_{0} T} f_{2} \equiv e^{k_{0} T} f_{2}, \quad w(0)=0,
$$

with $\tilde{A}$ defined in terms of $\tilde{Q}(t)$ and $\tilde{k}_{0}$.

From (4.28), (4.51) and (4.57), we get

$$
\begin{aligned}
\left\|u(t)-w_{\mathrm{app}}(t) \exp \left\{-\left[k_{0}+\frac{\gamma(t-T)}{2}\right] t\right\}\right\|_{2} \\
\leqq M^{(T-t) / T} \delta^{t / T} \exp \left\{\frac{\gamma t(T-t)}{2}\right\}+O\left(\Delta t^{2}\right),
\end{aligned}
$$

where $u(t)$ is any solution of (4.35) satisfying (4.2) and (4.3). From (4.60), we may observe the effect of the extra smoothing of Case 2. The uncertainty in the solutions has been increased by a factor $\exp \{\gamma t(T-t) / 2\}$ over that in Case 1 , where $\gamma$ is the upper bound on $\partial c / \partial t$.

We now remark that, in both Cases 1 and 2, the uncertainty is less than would have been predicted by the Agmon-Nirenberg convexity result (4.7). This is because the latter is of general validity. As a matter of fact, the error bound obtained via (4.7) is what our method yields for Case 3, the case of most severe smoothing of the initial data. To analyze Case 3, we will reduce it to Case 1 by stretching the time variable. This stretching transformation was shown to us by Joel Spruck.

In view of the transformation (4.51), it is sufficient to consider the case where

$$
\partial a / \partial t \leqq \alpha, \quad \alpha>0, \quad \partial c / \partial t \leqq 0 .
$$

With $a_{0}$ as in (4.32), let $\varphi(s)$ be the function

$$
\varphi(s)=\frac{a_{0}}{\alpha} \log \left(1+\frac{\alpha}{a_{0}} s\right), \quad s \geqq 0 .
$$

Then,

$$
\begin{aligned}
\varphi^{\prime}(s) & =a_{0} /\left(a_{0}+\alpha s\right)>0, \\
\varphi^{\prime \prime}(s) & =-a_{0} \alpha /\left(a_{0}+\alpha s\right)^{2}<0 .
\end{aligned}
$$

We shall put $t=\varphi(s)$ in (4.29). Let

$$
\begin{aligned}
& p(x, s)=u(x, \varphi(s)), \quad s \geqq 0, \\
& b(x, s)=a(x, \varphi(s)) \varphi^{\prime}(s), \\
& d(x, s)=c(x, \varphi(s)) \varphi^{\prime}(s) .
\end{aligned}
$$

Then, $p(x, s)$ satisfies

$$
\begin{aligned}
p_{s} & =\left[b(x, s) p_{x}\right]_{x}-d(x, s) p, & & 0<x<1, s>0, \\
p(x, 0) & \equiv u(x, 0)=f_{1}(x), & & 0 \leqq x \leqq 1, \\
p(0, s) & =p(1, s)=0, & & s \geqq 0 .
\end{aligned}
$$

From (4.32) and (4.61)-(4.64) we have

$$
\begin{array}{cl}
b(x, s) & >0, \quad d(x, s) \geqq 0, \\
b_{s} \leqq 0, & d_{s} \leqq 0 .
\end{array}
$$


Therefore, the transformation (4.62) reduces Case 3 to Case 1. Consider now the backward problem for the transformed equation (4.68). Let $f_{2}(x)$ be the given approximation to the data at time $T$ for (4.29). Then, $f_{2}(x)$ becomes terminal data for (4.68) at time $S$ where

$$
S=\left(a_{0} / \alpha\right)\left[e^{\alpha T / a_{0}}-1\right]
$$

Putting

$$
k_{0}=(1 / S) \log (M / \delta),
$$

we solve the auxiliary problem associated with $(4.68)$ on $[0, S]$; that is,

$$
\begin{aligned}
q_{s s}-A(s) q & =0, & & 0<s<s, \\
q(0) & =0, & & q(S)=e^{k_{0} s} f_{2},
\end{aligned}
$$

where $A(s)$ is constructed in the usual way, from $k_{0}$ and the right-hand side of (4.68). If $q_{\mathrm{apD}}(s)$ is the solution of the difference equations for (4.75), (4.76), it follows as in Case 1 that

$$
\left\|p(s)-q_{\mathrm{apD}}(s) e^{-k_{0} s}\right\|_{2} \leqq M^{(s-s) / s} \delta^{s / s}+O\left(\Delta s^{2}\right) .
$$

It is instructive to transform back to the unstretched time $t$. Let

$$
\varphi^{-1}(t)=\left(a_{0} / \alpha\right)\left(e^{\alpha t / a_{0}}-1\right)
$$

and define

$$
w_{\mathrm{app}}(t)=q_{\mathrm{app}}\left(\varphi^{-1}(t)\right), \quad 0<t<T .
$$

Put

$$
\mu(t)=\left(e^{\alpha T / a_{0}}-1\right) /\left(e^{\alpha t / a_{0}}-1\right) .
$$

Let $u(t)$ be any solution of (4.35) satisfying (4.2) and (4.3). From (4.71), (4.79), (4.80), we then have

$$
\left\|u(t)-e^{-k_{0} \varphi^{-1}(t)} w_{\mathrm{app}}(t)\right\|_{2} \leqq M^{(\mu-1) / \mu} \delta^{1 / \mu}+O\left(\Delta t^{2}\right),
$$

where $k_{0}$ is defined in (4.74). Thus, the uncertainty in (4.81) is that obtained from (4.7) with $m=0$ and $c=\alpha / a_{0}$.

The above discussion of the one-dimensional problem can be generalized. To begin with, the distinction between the three cases and the transformations (4.51) and (4.65)-(4.67) apply equally well to the problem in more than one space dimension

$$
\begin{gathered}
u_{t}=\nabla \cdot a(x, t) \nabla u-c(x, t) u, \quad x \in \Omega, t>0, \\
u=0 \text { on } \partial \Omega,
\end{gathered}
$$

where $a(x, t) \geqq a_{0}>0$ and $c(x, t) \geqq 0$. In fact, all of the ideas remain valid for higherorder parabolic problems which can be built up from (4.82), (4.83) such as

$$
u_{t}=(-1)^{s-1} Q^{s}(t) u, \quad t>0,
$$

where $s$ is a positive integer and where $Q(t)$ is the operator corresponding to the spatial part of (4.82) and the boundary conditions (4.83). Moreover, the fully discrete scheme of Section 3 can be generalized to apply to (4.84) while preserving all the 
necessary inequalities. In practice, because of the resulting algebraic problem, one will seldom be able to compute problems in more than two space dimensions in general domains, and with time-dependent coefficients.

To complete the discussion of the time-dependent case, we now show how the preceding ideas can be generalized so as to apply to the abstract " $V$-parabolic" problems of Section 3. Because of the level of generality, the discussion will now have to be restricted to the semidiscrete "method of lines" of Section 2.

Let $V$ be a closed subspace of the Sobolev space $H^{m}(\Omega)$ with the property that

$$
H_{0}^{m}(\Omega) \subset V \subset H^{m}(\Omega)
$$

and let $\|(\cdot)\|_{m}$ denote the norm on $H^{m}(\Omega)$. For each $t \geqq 0$, let $a(t ; u, v)$ be the symmetric bilinear form on $V$ given by

$$
a(t ; u, v)=\sum_{|p|,|a| \leqq m} \int_{\Omega} a_{p q}(x, t) D^{a} u \overline{D^{p} v} d x,
$$

where the $a_{p q}$ depend smoothly on $x$ and $t$ and

$$
a_{p q}=\bar{a}_{a p} \text {. }
$$

We assume $a(t ; u, v)$ to be strongly coercive on $V$, uniformly in $t$; this means that there exists a positive constant $\omega$, independent of $t$, such that

$$
a(t ; v, v) \geqq \omega\|v\|_{m}^{2} \quad \forall v \in V .
$$

Let $\dot{a}_{p q}$ denote $\partial a_{p q} / \partial t$ and define $\dot{a}(t ; u, v)$ to be the bilinear form on $V$ obtained from (4.86) when $a_{p q}$ is replaced by $\dot{a}_{\nu q}$. The form $\dot{a}(t ; u, v)$ will play an important role in the subsequent discussion. Both $a(t ; u, v)$ and $\dot{a}(t ; u, v)$ are continuous on $V \times V$. Thus, there exist constants $K_{1}>0$ and $K_{2} \geqq 0$ such that

$$
\begin{array}{ll}
|a(t ; u, v)| \leqq K_{1}\|u\|_{m}\|v\|_{m} & \forall u, v \in V, \\
|\dot{a}(t ; u, v)| \leqq K_{2}\|u\|_{m}\|v\|_{m} & \forall u, v \in V .
\end{array}
$$

The form (4.86) generates the selfadjoint parabolic boundary problem

$$
\begin{gathered}
u_{t}=-P_{0}(t) u=-\sum_{|p|,|q| \leqq m}(-1)^{|p|} D^{p}\left(a_{p q}(x, t) D^{q} u\right), \quad x \in \Omega, t>0 . \\
B[u]=0, \quad x \in \partial \Omega, t \geqq 0 .
\end{gathered}
$$

We assume (4.86) to be such that the boundary conditions (4.92) are independent of $t$. Hence, under sufficient smoothness,

$$
\frac{\partial}{\partial t} B[u]=B\left[u_{t}\right]=B\left[P_{0} u\right]=0, \quad x \in \partial \Omega, t \geqq 0 .
$$

The backward beam equation associated with (4.91), (4.92) is

$$
\begin{aligned}
u_{t t} & =\left[P_{0}^{2}-\dot{P}_{0}\right] u, & & x \in \Omega, t>0, \\
B[u] & =B\left[P_{0} u\right]=0, & & x \in \partial \Omega, t \geqq 0 .
\end{aligned}
$$

According to Theorem 2, this is of the form $u_{t t}-A(t) u=0$, with $A(t)$ quasi $m$ accretive in $L^{2}(\Omega)$. Corresponding to the three cases in problem (4.29), we now make the following definition: 
Definition. The parabolic boundary problem (4.91), (4.92) is minimal-smoothing on $[0, T]$ if

$$
\dot{a}(t ; v, v) \leqq 0 \quad \forall v \in V, 0<t \leqq T .
$$

It is strongly-smoothing if

$$
\dot{a}(t ; v, v) \leqq \gamma\|v\|_{H}^{2}, \quad v \in V, 0<t \leqq T,
$$

where $\|(\cdot)\|_{H}$ is the norm on $L^{2}(\Omega)$ and $\gamma>0$. If, for some positive $\alpha$, we have

$$
\dot{a}(t ; v, v) \leqq \alpha\|v\|_{m}^{2}, \quad v \in V, 0<t \leqq T,
$$

the problem (4.91), (4.92) is said to be maximal-smoothing on [0,T].

We may write (4.94), (4.95) as

$$
u_{t t}=\left[P^{2}(t)-\dot{P}(t)\right] u,
$$

where $P^{2}(t)$ is the positive selfadjoint operator in $L^{2}(\Omega)$ defined by $P_{0}^{2}(t)$ and the boundary conditions $(4.95)$, and $\dot{P}(t)$ is the symmetric operator in $L^{2}(\Omega)$ corresponding to $\dot{P}_{0}(t)$ restricted to the domain of $P^{2}(t)$. Since

$$
\langle\dot{P}(t) v, v\rangle=\dot{a}(t ; v, v) \quad \forall v \in D_{P^{2}}(t),
$$

the inequalities (4.96)-(4.98) can be translated in terms of the operator $\dot{P}$.

With the above machinery, it is now easy to discuss the backward problem for (4.91), (4.92). Consider first the minimally-smoothing case. In this case, we choose $k_{0}$ as in (4.27) and use the difference scheme (2.10), (2.11) to solve the auxiliary problem

$$
\begin{gathered}
w_{t t}=\left[\left(P-k_{0}\right)^{2}-\dot{P}\right] w, \quad 0<t<T . \\
w(0)=0, \quad w(T)=e^{k_{0} T} f_{2},
\end{gathered}
$$

where $f_{2}$ is the given approximation to the terminal data. This leads to the estimate (4.28) as in the case of time-independent coefficients. In the strongly-smoothing case, we have

$$
\langle\dot{P}(t) v, v\rangle \leqq \gamma\langle v, v\rangle \quad \forall v \in D_{P^{2}}(t) .
$$

Applying the preliminary transformation (4.51) to (4.91), we may reduce the strongly smoothing case to the minimal case. With $\tilde{k}_{0}$ as in (4.56), this leads to the estimate (4.60). In the maximal-smoothing case, we first observe that the idea behind the stretching transformation (4.62) can be abstracted to the present problem. With $\omega$ and $\alpha$ the constants in (4.88) and (4.98), define the function

$$
\psi(s)=(\omega / \alpha) \log (1+\alpha s / \omega), \quad s \geqq 0 .
$$

Then, $\psi^{\prime}(s)>0, \psi^{\prime \prime}(s)<0$ and

$$
\omega \psi^{\prime \prime}+\alpha\left(\psi^{\prime}\right)^{2}=0 .
$$

From the bilinear form $a(t ; u, v)$ of $(4.86)$, we construct the form $b(s ; u, v)$ where

$$
\begin{aligned}
b(s ; u, v) & =a(\psi(s) ; u, v) \psi^{\prime}(s), \quad s \geqq 0, \\
& =\sum_{|p|,|q| \leqq m} \int_{\Omega} b_{p q}(x, s) D^{a} u \overline{D^{p} v} d x,
\end{aligned}
$$


with

$$
b_{p q}(x, s)=a_{p q}(x, \psi(s)) \psi^{\prime}(s), \quad s \geqq 0 .
$$

Let $b^{\prime}(s ; u, v)$ be the symmetric bilinear form on $V$ obtained from (4.106) by replacing $b_{p q}(x, s)$ with $\partial b_{p q} / \partial s$. We then have

$$
b^{\prime}(s ; u, v)=\psi^{\prime \prime} a(\psi(s) ; u, v)+\left(\psi^{\prime}\right)^{2} \dot{a}(\psi(s) ; u, v) .
$$

Hence, using (4.88), (4.98) and (4.104),

$$
b^{\prime}(s ; v, v) \leqq\left[\alpha\left(\psi^{\prime}\right)^{2}+\omega \psi^{\prime \prime}\right]\|v\|_{m}^{2}=0 \quad \forall v \in V .
$$

We now put $t=\psi(s)$ in (4.91). Let

$$
\xi(x, s)=u(x, \psi(s)), \quad x \in \Omega, s \geqq 0 .
$$

Then $\xi$ satisfies the parabolic boundary problem

$$
\begin{aligned}
\xi_{s} & =-G_{0}(s) \xi, & & x \in \Omega, s>0, \\
B[\xi] & =0, & & x \in \partial \Omega, s \geqq 0,
\end{aligned}
$$

where $G_{0}(s)=\psi^{\prime}(s) P_{0}(\psi(s))$. This is the problem generated by $b(s ; u, v)$. Since $b^{\prime}(s ; v, v) \leqq 0$, we have the minimal-smoothing case for the transformed problem (4.111), (4.112). Proceeding as in the discussion of the one-dimensional problem (4.35), we get an estimate like (4.81) for the maximal smoothing case with $a_{0}$ replaced by $\omega$ in (4.80).

5. An Example. Consider the one-dimensional problem

$$
\begin{aligned}
u_{t} & =u_{x x}, & & 0<x<\pi, t>0, \\
u(0, t) & =u(\pi, t)=0, & & t \geqq 0, \\
u(x, 0) & =e^{-10} \operatorname{Sin} x+\operatorname{Sin} 2 x, & & 0 \leqq x \leqq \pi .
\end{aligned}
$$

This problem has the exact solution

$$
u(x, t)=e^{-(10+t)} \operatorname{Sin} x+e^{-4 t} \operatorname{Sin} 2 x .
$$

The particular initial data (5.3) was selected so as to generate a solution whose character changes with time. From (5.4), we see that, at $t=0$, the second harmonic dominates the solution with an amplitude of about $10^{5}$ times that of the first harmonic. At $t=10 / 3$, the two amplitudes are equal, and thereafter the first harmonic dominates. At $T=5$, the amplitude of the first harmonic is about 150 times that of the second. To illustrate our theory, we have computed this problem backwards in time, given the exact solution at $T=5$. Thus, the only error in the terminal data is that due to round-off in the machine (see below). To get an idea of how long a time span is physically represented by choosing $T=5$, we mention that in the numerical computation of forward parabolic problems via marching procedures, a "long time" is often taken to be about the half-life of the transient term [26]. In the above example, computing backwards from $T=5$ to near $t=0$ amounts to resurrecting the second harmonic after it has decayed by some eight or nine orders of magnitude! This is because at the early times, the solution consists almost entirely of the second harmonic. Needless to say, such long time backward calculations are hardly possible in physical 
problems where the terminal data are obtained from measurements with low accuracy. The point of the experiment is to illustrate the possibility of going from the smooth to the less smooth.

Following the development of Section 4.1, we are led to the auxiliary problem

$$
\begin{aligned}
w_{t \imath} & =w_{x x x x}+2 k w_{x x}+k^{2} w, & & 0<x<\pi, 0<t \leqq 5, \\
w(0, t) & =w_{x x}(0, t)=w(\pi, t)=w_{x x}(\pi, t)=0, & & 0 \leqq t \leqq 5, \\
w(x, 0) & =0, w(x, 5)=e^{5 k} f_{2}(x), & & 0 \leqq x \leqq \pi .
\end{aligned}
$$

Here $k$ is a positive number to be determined from (4.27). The computations were carried out in single-precision on a CDC 6600 at the Los Alamos Scientific Laboratories. Hence, $f_{2}(x)$ in (5.7) differs from (5.4) at $t=5$ by an amount $\delta$, where

$$
\delta \approx e^{-15} \times 10^{-14} \approx 10^{-20} .
$$

Using $M \approx 1$, we get $k_{0}=9.2$.

To approximate the spatial operator in (5.5) and the boundary conditions (5.6), we used the scheme of Section 3.1. In the present one-dimensional time-independent case, this reduces to the pentadiagonal $J \times J$ matrix $(H-k I)^{2}$, where $I$ is the identity and $H$ is the $J \times J$ tridiagonal matrix

$$
H=\frac{1}{\Delta x^{2}}\{-1,2,-1\}, \quad \Delta x=\frac{\pi}{J+1} .
$$

Using the natural ordering along lines $t=$ constant, the fully discrete scheme for (5.5)-(5.7) may be written as a block tridiagonal system of linear equations. The matrix of this system is precisely the matrix $Q$ in (2.19) with the $A^{n}$ 's replaced by $(H-k I)^{2}$. We chose $\Delta t=1 / 50$ and $\Delta x=\pi / 40$. The resulting system of order 9,711 was solved using the method of matrix decomposition described in [2]. Time of computation on the CDC 6600 was 4.5 seconds, even though the code incorporated provisions for inhomogeneous terms in (5.1)-(5.3).

Equation (4.28) bounds the $L^{2}$ norm of the absolute error. However, since the solutions of (5.1) decay exponentially, it is the relative error which is significant. In our example, one can estimate the quantity $\sigma(t)$ of (4.24) by using (4.28) and the known behavior of the solution (5.4). Making provision for truncation error and using $M=1, \delta=10^{-20}$, we conclude that a relative error of 10 percent or less should be attainable as far back as 90 percent of the way from $T=5$. Clearly, in view of the fundamental uncertainty (4.17), one cannot expect good results further back in time.

Two computations of (5.5)-(5.7) were performed, one with $k=k_{0}=9.2$ and the other with $k=12$. Only the computation with $k=12$ is displayed. In Table 1 , the function $\sigma(t)$ of (4.24) is tabulated. We see that $\sigma$ is less than or equal to 10 percent as far as 94 percent of the way back from $T=5$, and less than 3 percent as far as 89 percent of the way back. Since all our estimates are in terms of $L^{2}$ norms, one may ask whether the pointwise structure of the solutions is actually being computed. Figure 1 displays the computed evolution backwards in time of the terminal data, together with the absolute error (E). The exact solution (5.4), can be obtained by adding the curve (E) to the curve $(*)$. We have good agreement up to $t=.3$. At $t=.02$, the relative error is about 73 percent. It is interesting, but very likely fortuitous, that the qualitative behavior of the solution is still preserved at $t=.02$. 


\begin{tabular}{|c|c|c|c|c|c|c|c|c|c|}
\hline $\mathbf{T}$ & NORM(RE) & $\mathbf{T}$ & NORM(RE) & $\mathbf{T}$ & NORM(RE) & $\mathbf{T}$ & NORM(RE) & $\mathbf{T}$ & NORM(RE) \\
\hline .02 & 7.28E- 01 & 1.02 & 3.62E- 03 & 2.02 & 1.60E. 03 & 3.02 & 1.59E- 02 & 4.02 & 2.12E- 02 \\
\hline .04 & 5.35E- 01 & 1.04 & 3.32E- 03 & 2.04 & 1.65E- 03 & 3.04 & 1.65E- 02 & 4.04 & 2.08E- 02 \\
\hline 0.06 & 4.00E- 01 & 1.06 & 3.06E- 03 & 2.06 & 1.71E- 03 & 3.06 & 1.72E- 02 & 4.06 & 2.03E- 02 \\
\hline 0.08 & 3.09E- 01 & 1.08 & 2.82E- 03 & 2.08 & 1.77E- 03 & 3.08 & 1.79E- 02 & 4.08 & 1.99E- 02 \\
\hline 0.10 & 2.48E- 01 & 1.10 & 2.61E- 03 & 2.10 & $1.84 \mathrm{E} \cdot 03$ & 3.10 & $1.86 \mathrm{E}-02$ & 4.10 & 1.95E- 02 \\
\hline 0.12 & 2.09E- 01 & 1.12 & 2.42E- 03 & 2.12 & 1.91E- 03 & 3.12 & 1.93E- 02 & 4.12 & 1.91E- 02 \\
\hline .14 & $1.84 \mathrm{E}$ & 1.14 & 2.25E- 03 & 2.14 & 1.99E- 03 & 3.14 & 2.00 E- 02 & 4.14 & 1.86E- 02 \\
\hline 0.16 & $1.66 \mathrm{E}$ & 1.16 & 2.10E- 03 & 2.16 & 2.07E- 03 & 3.16 & 2.07E- 02 & 4.16 & E. 02 \\
\hline .18 & 1.53 & 1.18 & $1.97 \mathrm{E}$ & 2.18 & 2.10 & 3.18 & 2.14E- 02 & 4.18 & E. 02 \\
\hline 0.20 & & 1.20 & & 2.20 & $2.26 \mathrm{E}-03$ & 3.20 & $2.20 \mathrm{E}-02$ & 4.20 & 1.73E- 02 \\
\hline 0.22 & 1.33E- 01 & 1.22 & $1.75 E$ & 2.22 & 2.36E. 03 & 3.22 & 2.27E- 02 & 4.22 & $1.69 E-02$ \\
\hline .24 & $1.25 \mathrm{E}$ & 1.24 & $1.67 E$ & 2.24 & 2.47E- 03 & 3.24 & 2.33E- 02 & 4.24 & E. 02 \\
\hline 0.26 & 1.1 & 1.26 & & 2.26 & -03 & 3.26 & .02 & 4.26 & $=02$ \\
\hline 0.28 & 1.08 & 1.28 & 1.52 & 2.28 & 2.70E- 03 & 3.28 & 2.45E- 02 & 4.28 & -02 \\
\hline 0.30 & $1.00 \mathrm{E}$ & 1.30 & 1.47 & 2.30 & 2.83E- & 3.30 & & 4.30 & \\
\hline 0.32 & 9.2 & 1.32 & & 2.32 & 2.9 & 3.32 & .02 & 4.32 & -02 \\
\hline 0.34 & 8.53E- 02 & 1.34 & $1.38 \mathrm{E}$ & 2.34 & $3.11 \mathrm{E}-03$ & 3.34 & 2.60E- 02 & 4.34 & 02 \\
\hline 36 & & 1.36 & & 2.36 & 03 & 3.36 & 02 & 4.36 & .02 \\
\hline 38 & 7.21 & 1.38 & & 2.38 & 03 & 3.38 & 2.6 & 4.38 & 02 \\
\hline 40 & 6.62 & 1.40 & $1.28 \mathrm{E}$ & 2.40 & & & & & \\
\hline 0.42 & 6.0 & 1.42 & & 2.42 & 3.77 & 3.42 & 2.7 & 4.42 & 02 \\
\hline 0.44 & 5.54 & 1.44 & 1.24 & 2.44 & 3.96 & 3.44 & 2. & 4.44. & \\
\hline 0.46 & 5.06 & 1.46 & 1.22 & 2.46 & 4.1 & 3.46 & 02 & 4.46 & 02 \\
\hline 0.48 & 4.62 & 1.48 & 1.2 & 2.48 & 4.38 & 3.48 & & 4.48 & \\
\hline 0.50 & & 1.50 & & 2.50 & & 3.50 & & 4.50 & \\
\hline 0.52 & 3.84 & 1.52 & 1.19 & 2.52 & & 3.52 & 2.8 & 4.52 & 02 \\
\hline 0.54 & 3.50 & 1.54 & 1.18 & 2.54 & 5.08 & 3.54 & 2.8 & 4.54 & \\
\hline .56 & $3.18 \mathrm{E}$ & 1.56 & 1.18 & 2.56 & & 3.56 & 02 & 4.56 & 03 \\
\hline 58 & 2.89 & 1.58 & & 2.58 & & 3.58 & 02 & 4.58 & 03 \\
\hline 60 & 2.63E- 02 & 1.60 & 1.1 & 2.60 & 5.9 & 3.60 & & 4.60 & \\
\hline 62 & 2.39E- 02 & 1.62 & 1.17 & 2.62 & & 3.62 & 02 & 4.62 & .03 \\
\hline 64 & 2.17E- 02 & 1.64 & $1.18 E$ & 2.64 & 6.5 & 3.64 & 2.76 & 4.64 & .03 \\
\hline 66 & 1.97E- 02 & 1.66 & 1.18 & 2.66 & & 3.66 & 02 & 4.66 & .03 \\
\hline 68 & 1.79 & 1.68 & & 2.68 & & 3.68 & 2.7 & 4.68 & 03 \\
\hline 70 & 1.63E- 02 & 1.70 & 1.19E- 03 & 2.70 & 7.55 & 3.70 & 2.70 & 4.70 & \\
\hline 72 & 1.48E- 02 & 1.72 & $1.20 E-03$ & 2.72 & -03 & 3.72 & 02 & 4.72 & 03 \\
\hline 0.74 & 1.34E. 02 & 1.74 & 1.21 & 2.74 & 8.33 & 3.74 & 2.64 & 4.74 & 5.62 \\
\hline .76 & 1.22E- 02 & 1.76 & 1.22E- 03 & 2.76 & 8.75 & 3.76 & 2.6 & 4.76 & -03 \\
\hline 78 & 1.11E. 02 & 1.78 & & 2.78 & & 3.78 & & 4.78 & 4.75 \\
\hline 80 & 1.01E. 02 & 1.80 & 1.25E- 03 & 2.80 & 9.63E- 03 & 3.80 & 2.54E- 02 & 4.80 & 4.32E- 03 \\
\hline 82 & 9.14E-03 & 1.82 & 1.26E- 03 & 2.82 & 1.01E- 02 & 3.82 & 2.51 & 4.82 & 3.89 \\
\hline 84 & & 1.84 & & 2.84 & & 3.84 & 2.47E- 02 & 4.84 & 3.45 \\
\hline 86 & 7.55E- 03 & 1.86 & 1.31E- 03 & 2.86 & 1.11E. 02 & 3.86 & 2:44E- 02 & 4.86 & 3.02E- 03 \\
\hline 0.88 & 6.87E- 03 & 1.88 & 1.33E- 03 & 2.88 & 1.16E- 02 & 3.88 & 2.40E- 02 & 4.88 & $2.59 \mathrm{E}$ \\
\hline 0.90 & 6.25E- 03 & 1.90 & 1.36E- 03 & 2.90 & 1.22E- 02 & 3.90 & 2.36E- 02 & 4.90 & 2.16E- 03 \\
\hline 0.92 & 5.69E- 03 & 1.92 & 1.39E. 03 & 2.92 & 1.28E- 02 & 3.92 & 2.32E- 02 & 4.92 & 1.73 \\
\hline & & 1.94 & 1.43E- 03 & 2.94 & 1.33E- 02 & 3.94 & 2.28E- 02 & 4.94 & 1.29E- 03 \\
\hline 0.96 & 4.73E- 03 & 1.96 & $1.46 \mathrm{E} \cdot 03$ & 2.96 & 1.40 E- 02 & 3.96 & 2.24E- 02 & 4.96 & 8.62E- 04 \\
\hline & & 1.98 & & 2.98 & $1.46 \mathrm{E}-02$ & 3.98 & 2.20E- 02 & 4.98 & 4.31E- 04 \\
\hline 1.00 & 3.95E- 03 & 2.00 & 1.55E- 03 & 3.00 & 1.52E- 02 & 4.00 & 2.16E- 02 & 5.00 & 3.97E- 13 \\
\hline
\end{tabular}

Relative error in the $L^{2}$ norm as a function of time. 

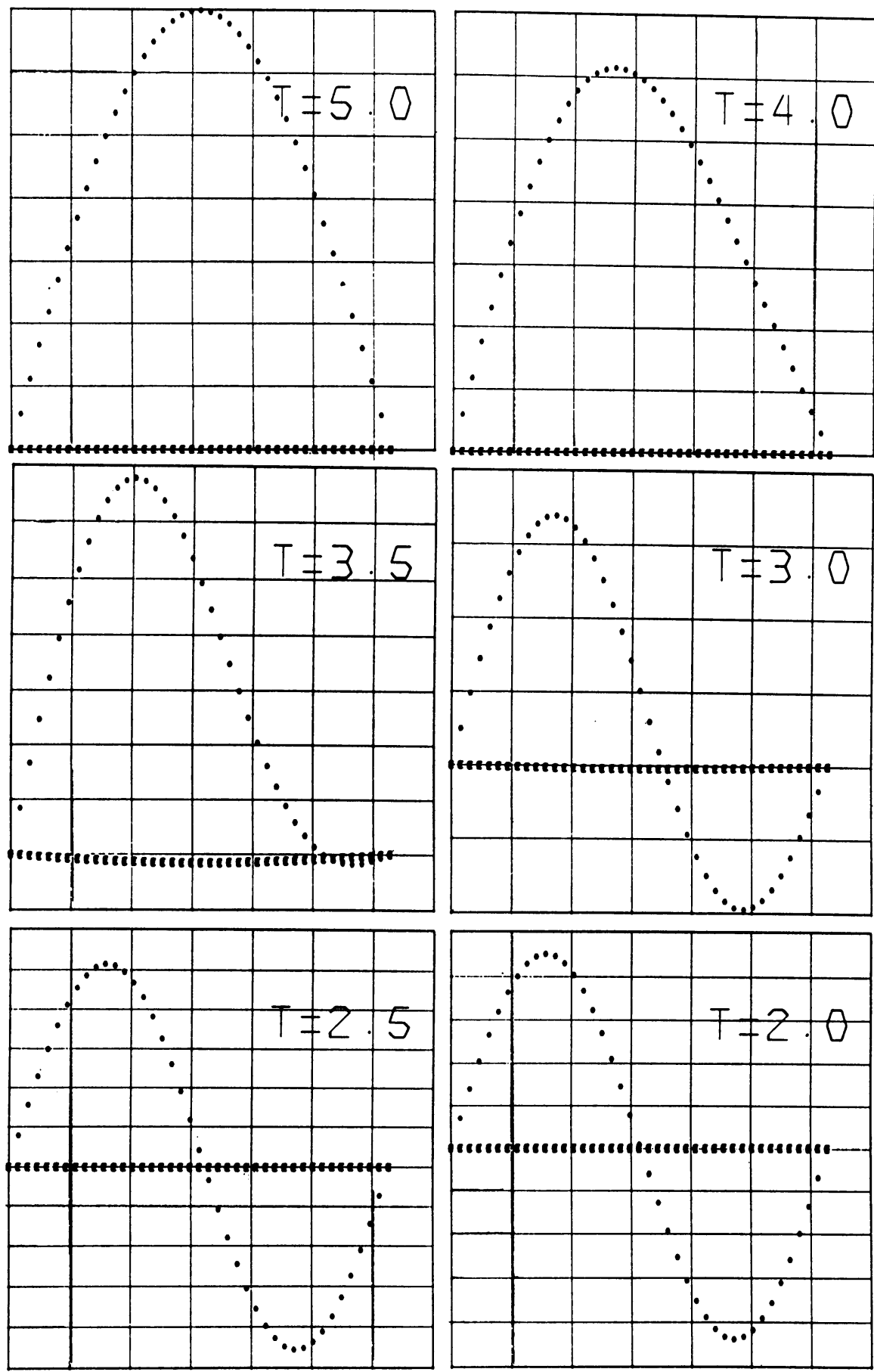

FIGURE 1

Backward evolution of slightly perturbed first harmonic in the presence of round-off error. (*) denotes computed solution, (E) absolute error. 

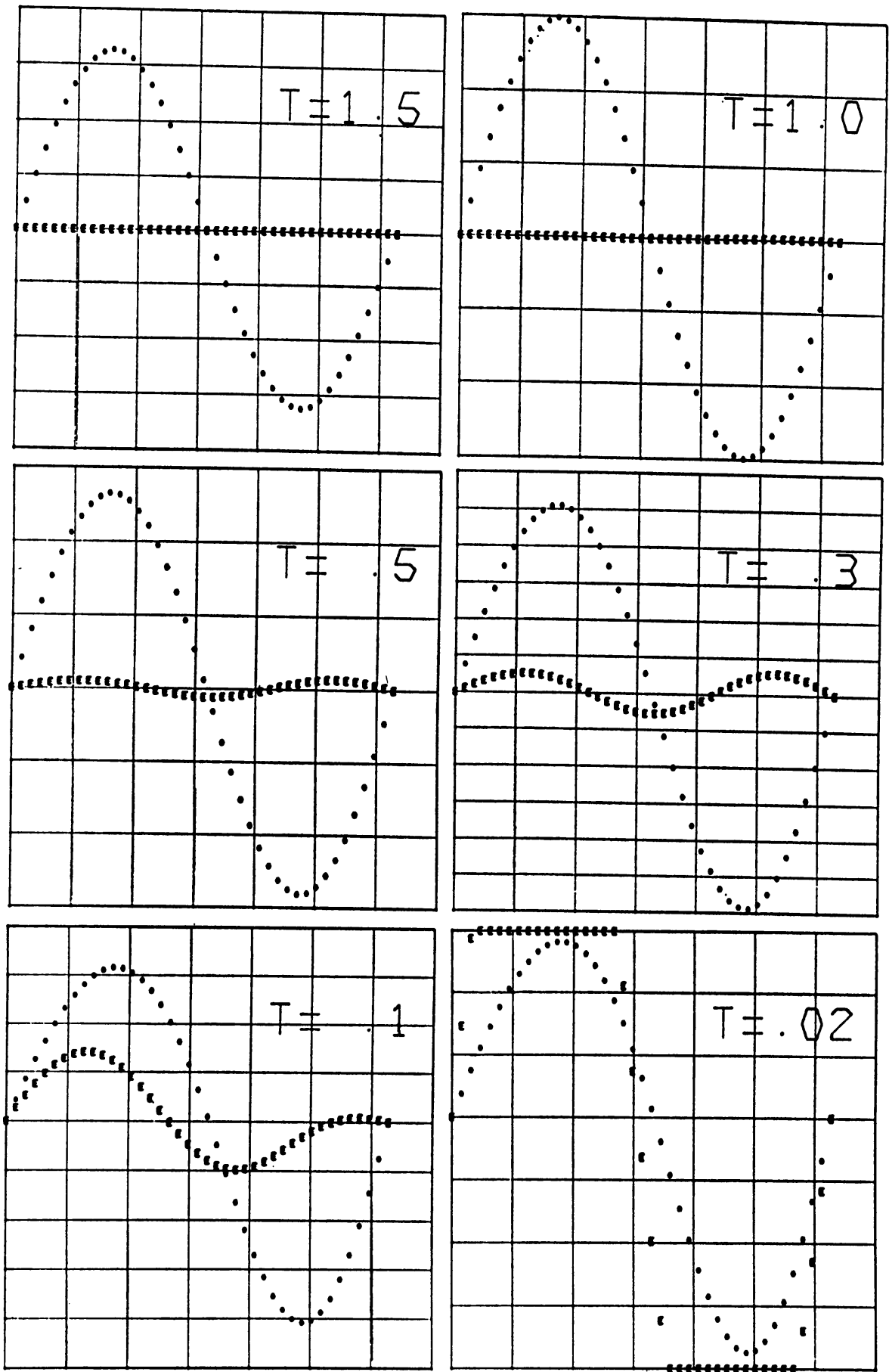

FIGURE 1 (Continued) 
The computation with $k_{0}=9.2$ produced similar results. Although the interval of 10 percent relative error was essentially the same as with $k=12$, the relative errors were generally larger. The reason for this is the following. With $k_{0}=9.2$, the matrix $\left(H-k_{0} I\right)^{2}$ is nearly singular, since 9 is an eigenvalue of the spatial operator in (5.1)-(5.2). From Theorem 1 of Section 2 , with $\beta=0$, we see that we have linear decay of the data, and hence of the errors. On the other hand, $k=12$ lies midway between the third and fourth eigenvalues of the differential operator, and this leads to a positive-definite matrix $(H-k I)^{2}$. The resulting exponential decay of the errors, due to the hyperbolic sines, accounts for the better results.

University of California

Los Alamos Scientific Laboratory

Los Alamos, New Mexico 87544

Department of Mathematics and Statistics

University of New Mexico

Albuquerque, New Mexico 87106

1. R. S. ANDERSSEN, A Review of Numerical Methods for Certain Improperly Posed Parabolic Partial Differential Equations, Technical Report No. 36, Computer Centre, Australian National University, August 1970.

2. B. L. Buzbee, G. H. Golub \& C. W. Nielson, "On direct methods for solving Poisson's equations," SIAM J. Numer. Anal., v. 7, 1970, pp. 627-656.

3. J. R. CANnon, Some Numerical Results for the Solution of the Heat Equation Backwards in Time, Proc. Adv. Sympos. Numerical Solutions of Nonlinear Differential Equations (Madison, Wis., 1966), Wiley, New York, 1966, pp. 21-54. MR 34 \#7037.

4. J. R. Cannon \& J. Douglas, JR., The Approximation of Harmonic and Parabolic Functions on Half-Spaces From Interior Data, Numerical Analysis of Partial Differential Equations (CIME 2० Ciclo, Ispra, 1967), Edizioni Cremonese, Rome, 1968, pp. 193-230. MR 39 \# 5076.

5. A. CARASSo, "The abstract backward beam equation," SIAM J. Math. Anal., v. 2, 1971, pp. 193-212. MR 44 \#5636.

6. A. CARASSo, "The backward beam equation: Two $A$-stable schemes for parabolic problems," SIAM J. Numer. Anal., v. 9, 1972, pp. 406-434.

7. R. Courant, Methods of Mathematical Physics. Vol. II: Partial Differential Equations, Interscience, New York, 1962. MR 25 \#216.

8. J. Douglas, JR., Approximate Solution of Physically Unstable Problems, Ecole CEA-EDF, Paris, 1965.

9. J. Douglas, JR., The Approximate Solution of An Unstable Physical Problem Subject to Constraints, Functional Analysis and Optimization, Academic Press, New York, 1966, pp. 65-66. MR 35 \#6962.

10. J. Douglas, JR., Approximate Continuation of Harmonic and Parabolic Functions, Proc. Sympos. Numerical Solution of Partial Differential Equations (Univ. Maryland, 1965), Academic Press, New York, 1966, pp. 353-364. MR 34 \#2206. 1969.

11. A. Friedman, Partial Differential Equations, Holt, Rinehart and Winston, New York,

12. F. JoHN, "Numerical solution of the equation of heat conduction for preceding times," Ann. Mat. Pura Appl., v. 40, 1955, pp. 129-142. MR 19, 323.

13. T. Kato, Perturbation Theory for Linear Operators, Die Grundlehren der math. Wissenschaften, Band 132, Springer-Verlag, New York, 1966. MR 34 \#3324.

14. R. LATTÈs \& J.-L. Lions, Méthode de Quasi-Reversibilité et Applications, Travaux et Recherches Mathématiques, no. 15, Dunod, Paris, 1967. MR 38 \#874.

15. M. M. Lavrentiev, Some Improperly Posed Problems of Mathematical Physics, Springer Tracts in Natural Philosophy, vol. II, Springer-Verlag, New York, 1967.

16. M. M. LAVRENTIEV, "Numerical solution of conditionally properly posed problems," Numerical Solution of Partial Differential Equations. II (SYNSPADE 1970), B. Hubbard (Editor), Academic Press, New York, 1971.

17. M. LEes \& M. H. ProtTER, "Unique continuation for parabolic differential equations and inequalities," Duke Math. J., v. 28, 1961, pp. 369-382. MR 25 \#4254.

18. J. L. Lions \& B. Malgrange, "Sur l'unicité rétrograde dans les problèmes mixtes paraboliques," Math. Scand., v. 8, 1960, pp. 277-286. MR 25 \#269. 
19. J. L. Lıons, Equations Différentielles Opérationnelles et Problèmes aux Limites, Die Grundlehren der math. Wissenschaften, Band 111, Springer-Verlag, Berlin, 1961. MR 27 \#3935.

20. Y. L. Lure, The Special Functions and Their Approximations. Vols. 1, 2, Math. in Sci. and Engineering, vol. 53, Academic Press, New York, 1969. MR 39 \#3039; MR 40 \#2909.

21. K. Miller, "Three circle theorems in partial differential equations and applications to improperly posed problems," Arch. Rational Mech. Anal., v. 16, 1964, pp. 126-154. MR 29 \#1435.

22. L. E. Payne, On Some Non Well Posed Problems For Partial Differential Equations, Proc. Adv. Sympos. Numerical Solutions of Nonlinear Differential Equations (Madison, Wis., 1966), Wiley, New York, 1966, pp. 239-263. MR 35 \#4606.

23. C. PuccI, "Sui problemi di Cauchy non "ben posti"," Atti Accad. Naz. Lincei Rend. Cl. Sci. Fis. Mat. Natur., v. 18, 1955, pp. 473-477. MR 19, 426.

24. R. D. RichtMYER \& K. W. MORTON, Difference Methods for Initial-Value Problems, 2nd ed., Interscience Tracts in Pure and Appl. Math., no. 4, Interscience, New York, 1967. MR 36 \#3515.

25. R. SAYLOR, "Time reversal in abstract Cauchy problems," SIAM J. Math. Anal., v. 2, 1971, pp. 454-457.

26. R. S. VARGA, "Some results in approximation theory with applications to numerical analysis," Numerical Solution of Partial Differential Equations. II (SYNSPADE 1970), B. Hubbard (Editor), Academic Press, New York, 1971.

27. F. John, Partial Differential Equations, Math. Appl. to Physics, Springer, New York, 1970, pp. 229-315. MR 41 \#5752.

28. J. N. FrankLIN, "Well-posed stochastic extensions of ill-posed linear problems," J. Math. Anal. Appl., v. 31, 1970, pp. 682-716. MR 42 \#2556.

29. W. L. Miranker, "A well posed problem for the backward heat equation," Proc. Amer. Math. Soc., v. 12, 1961, pp. 243-247. MR 22 \#11216. 